https://helda.helsinki.fi

\title{
Radiolabeling of Theranostic Nanosystems
}

\section{Das, Sudeep}

Springer Nature Switzerland AG

2021-02-05

Das , S , Imlimthan , S , Airaksinen , A \& Sarparanta, M 2021, Radiolabeling of Theranostic Nanosystems . in F Fontana \& H A Santos (eds), Bio-Nanomedicine for Cancer Therapy . 1 edn , Advances in Experimental Medicine and Biology , vol. 1295 , Springer Nature Switzerland AG, Cham , pp. 49-76 . https://doi.org/10.1007/978-3-030-58174-9_3

http://hdl.handle.net/10138/340710

https://doi.org/10.1007/978-3-030-58174-9_3

acceptedVersion

Downloaded from Helda, University of Helsinki institutional repository.

This is an electronic reprint of the original article.

This reprint may differ from the original in pagination and typographic detail.

Please cite the original version. 


\begin{tabular}{|l|l|c|}
\hline Book title & Bio-nanomedicine for Cancer Therapy \\
\hline Chapter title & Radiolabeling of theranostic nanosystems \\
\hline Contributors & Sudeep Das, Ph.D. & Department of Chemistry \\
& Surachet Imlimthan, M.Sc. & Radiochemistry \\
& Anu J. Airaksinen, Ph.D. & University of Helsinki \\
& Mirkka Sarparanta, Ph.D. & Finland \\
\hline
\end{tabular}

\section{Introduction}

The term theranostic coined by the merging of the terms 'therapeutic' and 'diagnostic', refers to multifunctional nanosystems that are able to provide both therapeutic (e.g. delivery of a chemotherapeutic payload) and diagnostic (e.g. imaging or therapy monitoring) functions in the same platform. Radioisotopes can be employed in two ways for the development of theranostic nanosystems. First, the nanosystems can be radiolabeled with imagingcompliant isotopes such as those utilized for positron emission tomography (PET) or singlephoton emission computed tomography (SPECT), which emit gamma radiation that can be detected and quantified non-invasively outside of the subject using dedicated imaging systems. This will give information on the localization of the nanosystem in the body, including the targeting ability, kinetics of nanomaterial tumor accumulation, the route and rate of nanosystem elimination and circulation time. This information provides vital feedback on the performance of the nanosystem based on its structure-activity relationship, and guides future structural optimization for improved in vivo behavior. Additionally, when radiolabeled with diagnostic radioisotopes, the nanosystems can be used to screen for patients who will have sufficiently high nanosystem tumor accumulation to permit the attainment of therapeutically relevant payload concentrations in the tumor. In this way, the nanosystem itself can be used for patient stratification instead of a surrogate imaging biomarker. Second, the highly energetic particle radiation ( $\alpha, \beta^{-}$and Auger electrons) arising from radioactive decay of radioisotopes conjugated to the nanosystem, can be employed as a therapeutic strategy either alone or synergistically with chemotherapy. Due to their high molar activity, therapeutic radioisotopes are lucrative payloads for theranostic nanosystem development, as very small amounts of the radioisotope are often enough to impart therapeutically relevant radioactive doses at the tumor. This is in contrast to the feasibility limitations set by a limited drug loading degree in many cancer-targeting nanosystems under development today.

In this chapter, we will give an overview of current and emerging radiolabeling strategies for the radiolabeling of theranostic nanosystems either for imaging, radiotherapy, or combination of the two, as well provide a basic outline for the biological evaluation of radiolabeled theranostic nanosystems. Finally, a review of selected contemporary examples of preclinical and clinical studies utilizing radiolabeled theranostic nanosystems is given.

\section{Key concepts in radiochemistry for theranostic nanosystem development}

\subsection{Radioactive decay and properties of radiolabeled tracers}

Radioactive decay results from the loss of energy needed for an unstable nucleus to return to a stable state. This energy can be emitted in a number of ways ranging from photons 
(gamma radiation) to X-rays to particles ( $\alpha$ and $\beta^{-}$particles, positrons and Auger electrons), collectively known as ionizing radiation. The stable state can be attained either by a single decay emission (as for the isotope fluorine-18 $\left({ }^{18} \mathrm{~F}\right.$ in Figure 1a) or as a result of a decay chain, where the energy is lost in a series of decays through different daughter nuclei like in the case of the alpha emitter actinium-225 (225Ac, Figure 1b).

a

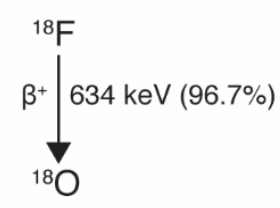

b $\quad{ }^{225} \mathrm{Ac}$

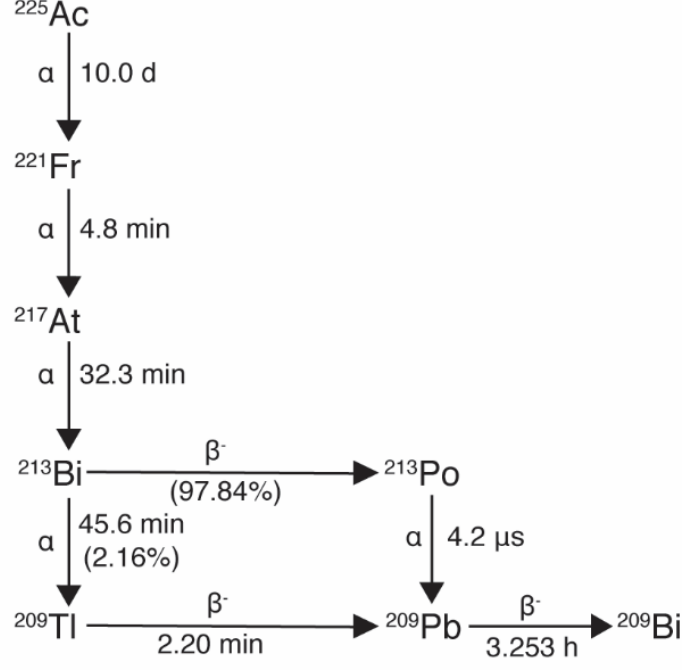

Figure 1. Radioactive decay can occur with a single emission or by multiple decays referred to as a "decay chain". The short-lived positron emitter ${ }^{18} \mathrm{~F}(\mathrm{~A})$ decays predominantly by positron emission (the rest is by electron capture), whereas heavy ${ }^{225} \mathrm{Ac}$ (B) decays by a series of alpha and beta decays through a number of daughter nuclei.

The fundamental premise to the use of radiolabeled compounds as tracers for the respective non-radiolabeled compounds is that radioactive isotopes behave chemically identically to the non-radioactive isotopes of the same element. The terms tracer, imaging probe, and imaging agent are used interchangeably in the literature and all refer to radiolabeled compounds used to track a structurally identical non-radiolabeled compound in vivo.

The most important properties of a radioisotope from the theranostic standpoint are the quality and energy of the emitted radiation, the physical half-life $\left(t_{1 / 2}\right)$ of the radioisotope and the chemical properties of the element in question. Highly tissue-penetrant gamma radiation is needed for non-invasive nuclear imaging, whereas particle radiation is needed for the deposition of energy into the tumor tissue through its interactions with the emitted charged particles. The amount of energy deposited in tissue is determined by the linear energy transfer (LET) value, and is a factor of the path length of the particle in the tissue and the energy of the radiation. Out of charged particles, Helium nuclei ( $\alpha$ particles) and Auger electrons have the highest LET values with the path length of mere fractions of a micrometer, while lighter electrons ( $\beta^{-}$particles) travel millimeters depending on their energy, and consequently have lower LET values as illustrated with an example of radioimmunoconjugates in Figure 2. The range of the particle radiation in tissue should be matched to the dimensions of the tumor to ensure the deposition of the energy in the tumor tissue as illustrated in Figure 3. 


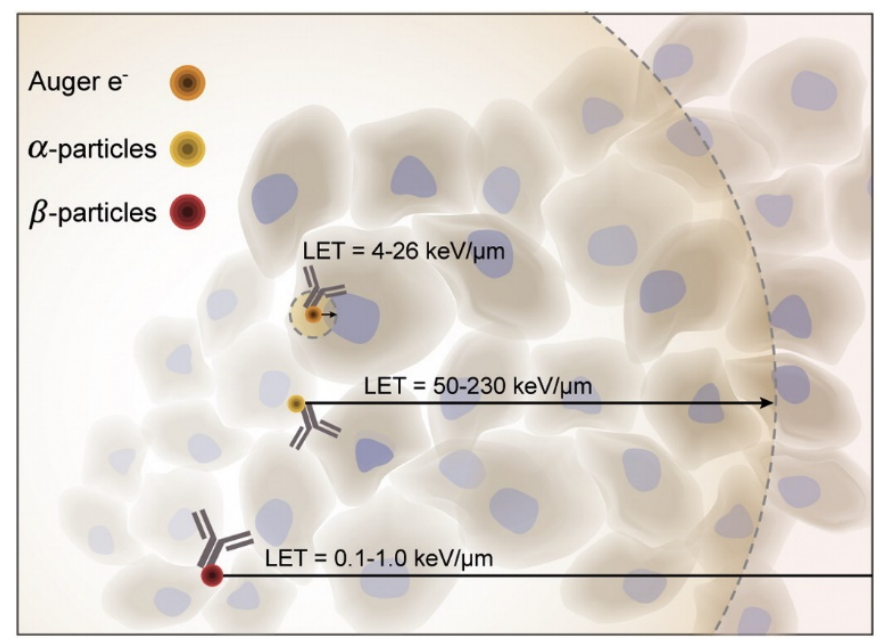

Figure 2. Illustration of the track of $\alpha$-particles, $\beta$-particles or Auger electrons emitted by radiolabeled monoclonal antibodies targeted to cancer cells. The short track length of $\alpha$-particles $(28-100 \mu \mathrm{m})$ and Auger electrons $(<0.5 \mu \mathrm{m})$ results in high linear energy transfer (LET) values of $50-230 \mathrm{keV} / \mu \mathrm{m}$ and 4 to $26 \mathrm{keV} / \mu \mathrm{m}$, respectively. $\beta$-Particles have a track length of $2-10 \mathrm{~mm}$ resulting in LET of $0.1-1.0 \mathrm{keV} / \mu \mathrm{m}$. The high LET of a-particles (50-100 $\mu \mathrm{m})$ and Auger electrons makes these forms of radiation more powerful for killing cancer cells than $\beta$-particles. Figure reprinted with permission from reference[1].
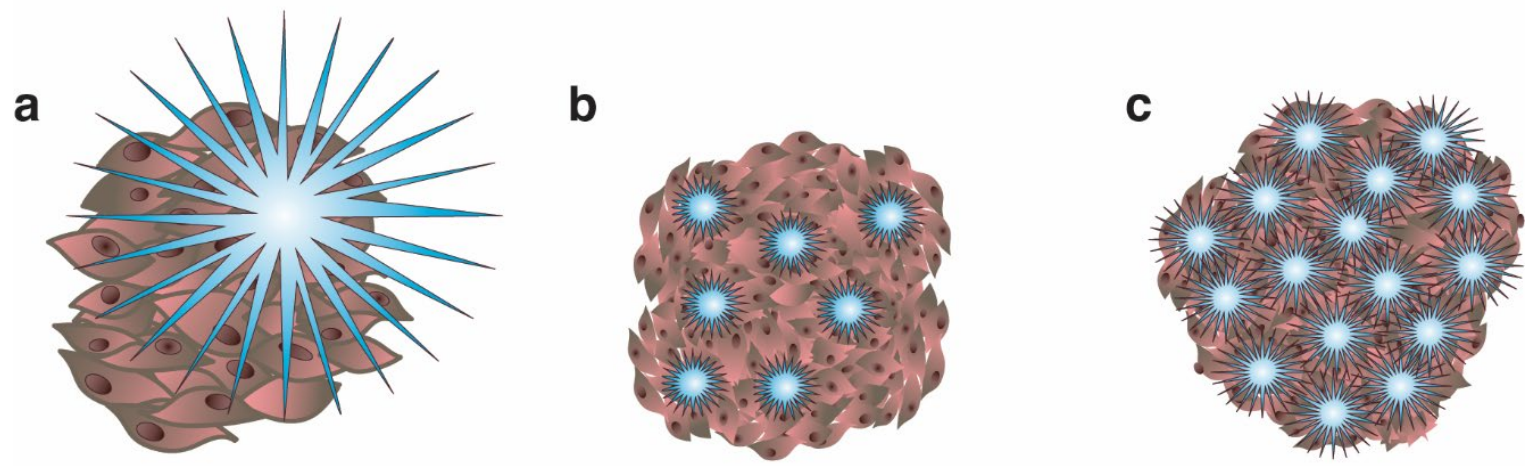

Figure 3. Matching the range of the radioactive emission to the dimensions of the target is important for the efficacy of the radiotherapy. Subpar effects will be seen in a small tumor treated with long-range (low LET) irradiation (A) or in a large mass treated with a short-range (high LET) irradiation with heterogenous distribution (B), whereas optimal results are achieved when a relatively homogenous distribution of an intermediate LET radioisotope is attained $(\mathrm{C})$. Figure adapted from reference [2].

The radionuclide half-life is determined by equation 1 , where

$t_{1 / 2}=\frac{\ln 2}{\lambda}$

where $\lambda$ is the decay constant for the radioisotope. The half-life tells us the amount of time required for the radioactivity $(A)$ of the material to decrease to one half of the original value $\left(A_{0}\right)$ and can be used to calculate the amount of radioactivity remaining in a sample at a given time $t$ as shown in Equation 2.

$A=A_{0} e^{-t / t_{1 / 2}}$ 
The half-lives of radioactive isotopes vary from mere milliseconds to thousands of years. For isotopes relevant for the development and radiolabeling of theranostic nanosystems the half-lives are most typically in the order of one hour to several days. This has a number of practical implications to the nanosystem development and choice of the radiolabeling strategy. First, the half-life of the isotope sets a practical limit to the maximum theoretical molar activity of the isotope that can be produced as shown in equation 3 , where $N$ is Avogadro's number.

molar activity $_{\max }=N \times \frac{\ln 2}{t_{1 / 2}}$

Second, as a guideline the radiosynthetic procedures and purification of the radiolabeled tracer should be completed within $\approx 2$ half-lives of the isotope to ensure meaningful molar activity (radioactivity per amount, usually expressed in gigabecquerels per micromole, GBq $\mu \mathrm{mol}^{-1}$ ) of the radiolabeled product for in vivo studies. Therefore, in order to obtain maximal molar activity radiolabels are best introduced late in the radiosynthesis, often achieved by the use of a prosthetic group, a radiolabeled precursor that can be conjugated to the nonradioactive target molecule or material using fast and selective chemistry preferably in a single step[3]. Third, the half-life of the radioisotope constrains the timeframe over which the passage of the radiolabeled compound can be tracked in vivo. For this purpose, the physical half-life of the radioisotope should be matched to the biological half-life of the vector, in this case the nanosystem. This is perhaps best illustrated by the case of radiolabeled antibodies, which have biological half-lifes in the order of days, therefore only longer-lived isotopes such as zirconium-89 $\left({ }^{89} \mathrm{Zr}, t_{1 / 2}=78.41 \mathrm{~h}\right)$, indium-111 $\left({ }^{111} \mathrm{In}, t_{1 / 2}=2.80 \mathrm{~d}\right)$, iodine-124 $\left({ }^{124} \mathrm{I}, t_{1 / 2}=4.18\right.$ d) and iodine-131 ( ${ }^{131} \mathrm{I}, t_{1 / 2}=8.03 \mathrm{~d}$ ) can be used for the tracking of directly labeled antibodies. Other important concepts in radiopharmaceutical development are the molar activity mentioned already earlier, specific activity, radiochemical yield, and radiochemical purity, the definitions of which will be reviewed next. The field of radiopharmaceutical chemistry follows a consensus nomenclature set forward in 2017 and compiled by Coenen et al.[4]

The molar activity of a radiolabeled compound is defined as the amount of radioactivity per mole of compound, whereas specific activity refers to the amount of radioactivity per mass, most often denoted in $\mathrm{GBq} \mathrm{mg}{ }^{-1}$ or $\mathrm{MBq} \mathrm{mg}^{-1}$. In most cases the exact molecular weight of the nanosystem is not known or reported, thus the specific activity gives a more meaningful denotion on how much radioactivity is incorporated to the nanosystem. Furthermore, if the final radiolabeled product contains non-radioactive starting material which cannot be removed, as is the case for most nanomaterials, the terms apparent molar activity and apparent specific activity should be used. The term radiochemical yield refers to the quantity (often expressed in percentage of the starting quantity) of the radioisotope incorporated to the radiolabeled product. Although analogous to chemical yield, an important notion of the radiochemical yield is that it needs to be always calculated using starting and final values for the same radioisotope which have been decay-corrected to the same point in time, typically for example the end of synthesis (EOS) or the end of bombardment (EOB) for the nuclear reaction producing the radioisotope. The term radiochemical purity in turn is analogous to chemical purity and is used to describe the purity of the product with respect to the presence of other radiolabeled compounds in the final product again decay-corrected to a fixed point in time. Typically, radiochemical purities exceeding $95 \%$ are considered adequate in terms of in vivo applications, but this level depends on the identity and 
pharmacokinetics of the impurity and more stringent criteria to radiochemical purity might need to be applied accordingly.

\subsection{Nuclear reactions for radionuclide production}

While some widely used short-lived radionuclides in clinic can be obtained by the elution from the relatively long-lived parent/daughter radionuclide pair generators, such as germanium-68/gallium-68 $\left({ }^{68} \mathrm{Ge} /{ }^{68} \mathrm{Ga}\right)$ and molybdenum-99/technetium-99m (99Mo/99mTc), most of the radionuclides in nuclear medicine are artificially manufactured through nuclear reactions of stable nuclides (Figure 4). The production of radionuclides is commonly carried out in either accelerators or reactors through nuclear reactions. The major components of the radioactive nuclide production are the target material, which can be in gaseous, liquid or solid form and the incident particles used for bombardment.

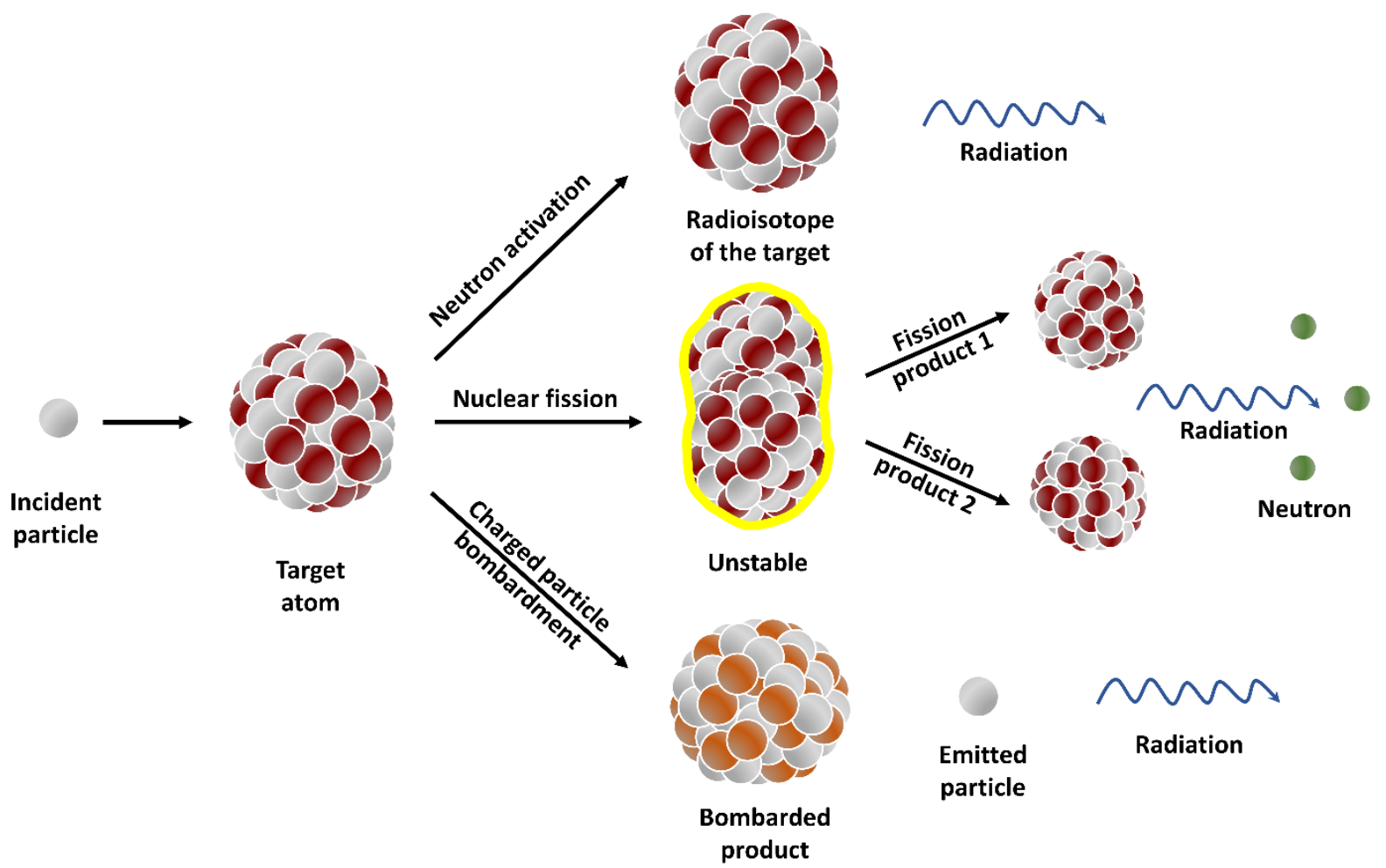

Figure 4. The most common nuclear reactions in radionuclide production. Neutron activation and nuclear fission are carried out in nuclear reactor while high-energy charged particle bombardment is carried out in accelerators.

In principle, the nuclear reaction occurs when the incident particle strikes through the nucleus of the target atom and excites it to an energy state to become unstable, resulting in the release of energy in the form of radiation or particle emissions. In high-energy charged particle bombardment, the nuclear reaction cannot take place unless the kinetic energy of an incident particle is high enough to overcome two energy barriers, which are the Coulomb barrier and Q-value for the reaction. When the high-energy particle collides with the nucleus of an atom, they combine and form a new compound nucleus, which is internally excited and subsequently decays by emitting radiation or nucleons (protons or neutrons). In general, any nuclear reaction can be represented by the following equation.

$$
x+A \rightarrow B+y+Q \text { or } A(x, y) B
$$


where $\mathrm{x}$ is the incident particle, $\mathrm{A}$ is the target, $\mathrm{B}$ is the product after the reaction, $\mathrm{y}$ is the emitted particle or radiation during the decay, and $Q$ is the reaction energy.

The reaction energy (Q-value) in nuclear reactions is the difference between the sum of the initial masses and the sum of the masses of the product nuclei. The cross-section $(\sigma)$ is one of the most important factors in nuclear reactions as it determines the total probability in the occurrence of the reaction under certain conditions (for example the incident particle energy and angle of observation). The cross-section can be expressed as the effective area where the incident particle interacts with the target nucleus: the larger the effective area, the greater the probability of the reaction. The cross-section has a dimension of an area $\left(\mathrm{cm}^{2}\right)$ and commonly expressed in the unit barn $\left(1 \mathrm{~b}=10^{-28} \mathrm{~m}^{2}\right.$ or $\left.10^{-24} \mathrm{~cm}^{2}\right)$. The value of the crosssection is variable and depended on many factors, such as the target nucleus, the incident particle energy, and the type of reaction.

In accelerators, radionuclides are produced by the bombardment of stable nucleus in the target with high-energy charged particles, such as protons $(p)$, deuterons $(d)$, alpha particles $(\alpha)$, and heavy ions, resulting in nuclear transformation of the stable nucleus to a different radionuclide, for example, production of yttrium-86 from strontium-86 $\left({ }^{86} \mathrm{Sr}(\mathrm{p}, \mathrm{n})^{86} \mathrm{Y}\right)$, indium111 from cadmium-112 $\left({ }^{112} \mathrm{Cd}(\mathrm{p}, 2 \mathrm{n}){ }^{111} \mathrm{In}\right)$, gallium-67 from zinc-67 $\left({ }^{67} \mathrm{Zn}(\mathrm{p}, \mathrm{n})^{67} \mathrm{Ga}\right)$, and fluorine-18 from oxygen-18 $\left({ }^{18} \mathrm{O}(\mathrm{p}, \mathrm{n}){ }^{18} \mathrm{~F}\right)$. Neutron activation and fission are the most common reactions in nuclear reactors. Neutron activation or $(n, y)$ reaction is a reaction where a stable target is bombarded with the thermal neutron flux in a nuclear reactor, for example technetium-99 from molybdenum-98 ( $\left.{ }^{98} \mathrm{Mo}(\mathrm{n}, \mathrm{y}){ }^{99} \mathrm{Tc}\right)$, strontium-89 from strontium$88\left({ }^{88} \mathrm{Sr}(\mathrm{n}, \mathrm{y}){ }^{89} \mathrm{Sr}\right)$, and lutetium-177 from lutetium-176 $\left({ }^{176} \mathrm{Lu}(\mathrm{n}, \mathrm{y}){ }^{177} \mathrm{Lu}\right)$. The neutronactivated product is an isotope of the target nuclide and has identical chemical properties. Also $(n, p)$ and $(n, \alpha)$ reactions can take place, as well as reactions proceeding through a short-lived intermediate product that decays to the desired radioisotope, such as the tellurium-131 ( $\left.{ }^{131} \mathrm{Te}\right)$ in ${ }^{130} \mathrm{Te}(\mathrm{n}, \mathrm{y}){ }^{131} \mathrm{Te}$, which decays by $\beta^{-}$emission to ${ }^{131} \mathrm{I}$. Nuclear fission is the most significant reaction in the nuclear reactor. The common targets in fission reactions are uranium-235 $\left({ }^{235} \mathrm{U}\right)$ and plutonium-239 $\left({ }^{239} \mathrm{Pu}\right)$. By adding an uncharged neutron to the bulky target nuclide, the compound nucleus becomes unstable and eventually splits into two or more smaller fragments with a number of free neutrons released. The most relevant fission products in nuclear medicine are ${ }^{131} \mathrm{I}$ for theranostic applications and ${ }^{99} \mathrm{Mo}$ for the use as radioactive parent in ${ }^{99} \mathrm{Mo} /{ }^{99 m} \mathrm{Tc}$ generators.

Radioisotope production is globally concentrated on facilities with accelerators and reactors. Smaller medical cyclotrons capable for the production of short-lived positron emitters $\left({ }^{11} \mathrm{C},{ }^{13} \mathrm{~N},{ }^{15} \mathrm{O},{ }^{18} \mathrm{~F},{ }^{89} \mathrm{Zr},{ }^{124} \mathrm{I}\right)$ are available at academic institutions and hospitals to account for the heavy use of these isotopes in the clinic and the fact that some of them cannot be transported due to the short half-life ( $<2$ hours). Also ${ }^{99} \mathrm{Mo} /{ }^{99 m} \mathrm{Tc}$ and ${ }^{68} \mathrm{Ge} /{ }^{68} \mathrm{Ga}$ generators are nowadays nearly ubiquitous in research and hospital laboratories because of their long usable life time which allows the users to dispense the radioisotope on demand. Longer-lived radioisotopes can be purchased through a number of vendors worldwide or acquired through academic collaborations in the case of more rare radioisotopes.

\subsection{Principles for the safe handling of radioactive materials}

Working safely with radioactivity requires a number of precautions and specialized working areas which are usually shared between many researchers at a given institute. The type of laboratory and shielding needed and the safe handling practices employed depends on the 
radioisotope and the amount of radioactivity that needs to be handled at one time. The type of the work also matters as some tasks like radiotracer administration to animals carry a greater risk of personnel exposure than for example the measurement of excised tissue samples in sealed tubes. Safe practices in working with radioactivity need to be followed at every step of the process from the radiolabeling synthesis to biological evaluation to disposal and storage of radioactive waste. The respective national and institutional regulations and guidelines are reviewed during introductory training on radiation safety and the radiation safety officer or department at the institute ensures personnel compliance by offering guidance and continued training. Dose monitoring needs to be offered to personnel working with radioactivity and medical clearance is most often needed prior to starting the work. As a general guideline, the amount of radiation dose accumulated during a procedure can be greatly reduced my using appropriate physical shielding (e.g. a fume hood with a lead-glass window or a remotely controlled synthesis apparatus), by increasing the distance from the radiation source (e.g. by handling radioactive vials with tongs or manipulators), and by minimizing the time spent handling the radioactive source (e.g. by careful planning of the procedure and practicing with mock runs with non-radioactive reagents). Additionally, the benefit gained using radioisotopes should always outweigh the risks associated with their use and the amount of radioactivity employed should be kept as low as reasonably possible.

\subsection{Radiometric detection methods and nuclear imaging}

The inherent properties of radioactivity contribute to the superior detection sensitivity and ease of quantitation for radiometric methods. First, as most of the radioisotopes employed in radiopharmaceutical development are rare or non-existent in natural systems as they have been artificially produced in nuclear reactions, there is no "natural" radioactive signal with the same energy spectrum that could hamper the analysis. Naturally abundant radioactive isotopes of elements such as potassium -40 (about $0.012 \%$ of natural potassium is ${ }^{40} \mathrm{~K}$ ) will of course be present in biological samples, but the levels are several orders of magnitude lower to any injected radiotracer and can be excluded from the analysis based on the spectra of the emitted radiation. Second, the aforementioned energy spectra allow for the identification of the desired radionuclide in a mixture of radionuclides and background radiation, enabling dual-isotope detection if the energies of the two isotopes are sufficiently different. Semiconductor gamma spectrometers of superior energy resolution are most often used in these applications. Finally, the number of radioactive disintegrations detected over a given time (count rate) is directly proportional to the number of radioactive atoms in the sample which greatly simplifies the quantification.

Quantification of radioactivity is the most straight-forward with radioisotopes emitting highly tissue-penetrant gamma radiation as this can be detected and quantified with minimal sample processing. The radioactivity content in excised tissue samples is for example determined simply by measuring the radioactivity in the freshly collected tissue using an automated gamma counter. The commercially available automated counters are the workhorses of both clinical and academic radiochemistry laboratories as they have a large sample handling capacity with automated sample changing, facile operation and robust welltype thallium-doped sodium iodide $\mathrm{Nal}(\mathrm{TI})$ detectors which usually require minimal maintenance over their lifetime. However, the Nal(TI) detectors are saturable, which means that the detector response remains linear only over a certain radioactive range and at higher count rates (more radioactive samples) the detector response deteriorates. Care must therefore be taken to maintain the sample activities at the linear range. Similarly, the sample radioactivity and counting geometry greatly affect the counting efficiency and dead time (the 
time for which the detector remains unresponsive and thus unable to detect the radiation) in semiconductor detectors and care must be taken to account for these factors in the quantification. Radioisotopes which do not emit any usable gamma radiation for detection purposes are rare especially in theranostic systems, but if needed liquid scintillation counting offers a convenient detection method for low-energy $\beta$ - and $\alpha$-emitting radioisotopes in biological samples. As the name implies, liquid scintillation counting relies on the detection of the light generated by the absorption of the energy emitted by the radioisotope in the scintillation medium, referred to as a cocktail. The liquid scintillation cocktails are commercially available solutions of solvents capable of absorbing the energy of the emitted radiation and organic scintillator molecules which in turn emit visible light in response to transfer of the energy from the solvent to the scintillator molecule. Unsurprisingly, energyrich aromatic ring structures and oxazoles compose the backbone for both solvent and scintillator molecules in commercial cocktails. The sample preparation for liquid scintillation counting is slightly more complicated than that for gamma counting, as the homogenized tissue samples need to be solubilized to the solvent and the sample rendered colorless by the addition of for example hydrogen peroxide. The sample composition, particularly color, can greatly affect the intensity of the detected light through quenching.

Nuclear imaging refers to the detection of gamma radiation emitted by a radioisotope outside of the living subject and subsequent process of reconstruction of the measured radiation to an image. For nuclear imaging in diagnostic purposes, the radioisotope must have gamma emissions, these can either be the two coincident 511-keV annihilation gammas of positron emitters, or single-energy gamma quanta emitted by other isotopes. The former are detected using a technique called positron emission tomography (PET) and the latter by single-photon emission computed tomography (SPECT). The principles of image formation in PET and SPECT imaging are outlined in Figure 5. Both PET and SPECT are fully translational imaging modalities, meaning that the tracer design, imaging system and setup are the same in small laboratory animals and in the clinic. The instrument design obviously varies as the systems are miniaturized for the imaging of laboratory rodents under inhalation anesthesia, but the detection principle and the study workflow remain the same. Since the molar activity of radiotracers is typically high, they can be administered in doses significantly lower than those used to elicit a pharmacological response. This makes it possible to use expensive and potentially harmful or even toxic substances in vivo without disturbance to the system under study. This microdosing principle[5] has made possible the investigational use of radiotracers early on in the drug development pipeline to study the target engagement and elimination of new drugs. For theranostic nanosystems the microdosing principle might not in all cases be directly applicable, as a macrodose of the nanomaterial might be needed for drug delivery. Nevertheless, the high molar activity of radioisotopes means that they can be used in the radiolabeling reactions in minute amounts and thus with minimal influence on the physicochemical properties of the nanosystem. A caveat in nuclear imaging modalities is the fact that they do not provide any anatomical information on the localization of the tracer except for what can be deduced from the tracer accumulation to non-target organs and knowledge on their location in the body. Therefore, co-registration of the nuclear image with an image obtained using another imaging modality, for example X-ray computed tomography (CT) or magnetic resonance imaging (MRI) is often necessary for the precise delineation of the sites of radiotracer accumulation. Conveniently, the large molecular weight of nanomaterials allows for the incorporation of both the radiolabel and a contrast agent for $\mathrm{CT}$ or MRI to be incorporated to the same construct, generating a multimodality tracer. 


\section{a}
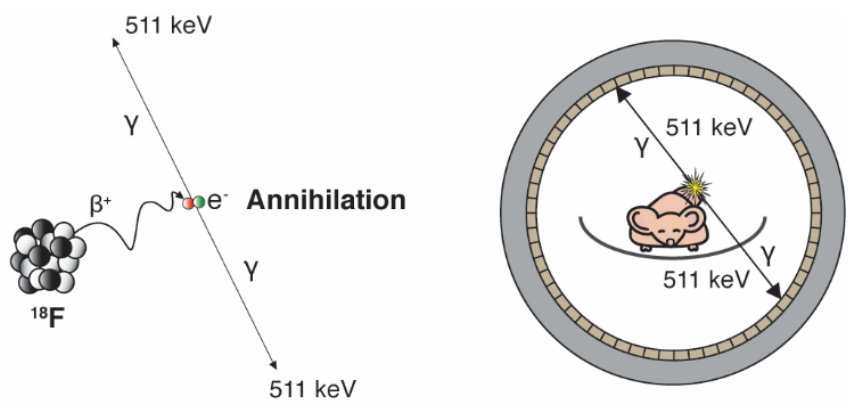

b

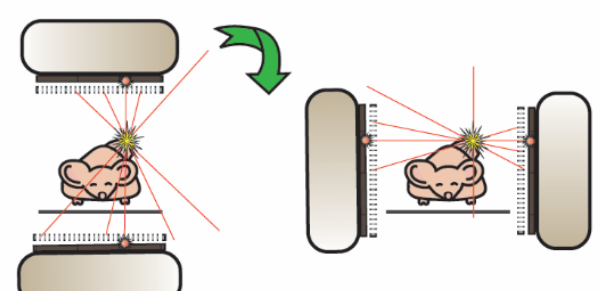

Figure 5. The principle of detection for positron emission tomography (PET) and single-photon emission computed tomography (SPECT). In PET (A), the detectors are arranged on a ring around the subject, and the coincident 511-keV gamma rays from the positron annihilation are detected. In SPECT (B), the tomographic image is generated by revolving an array of detectors and a collimator around the subject, and only the gamma quanta which are directly perpendicular to the collimator will pass through to the detectors for registration.

\section{Radiolabeling strategies}

\subsection{Radiometals}

Radiometals are radioactive isotopes of metal elements which have become an important tool for radiopharmaceutical drug development in nuclear medicine. Radiometal labels have been employed for a multitude of radiotracers from radiometal-labeled small molecules to peptides, antibodies and nanoparticles. Radiometal labeling strategies have several advantages over other radiolabeling techniques, for example the easy commercial availability of many radiometals, the pairs of diagnostic and therapeutic radionuclides of the same element, high specific activity, and in often cases favorable radiation dosimetry. The most relevant radiometal isotopes and their properties for SPECT, PET, and theranostics in the clinic and preclinical research have been listed in Table 1.

Table 1. Overview of radiometals for SPECT, PET and theranostics (data retrieved from the NuDat2 database, http://www.nndc.bnl.gov/nudat2)

\begin{tabular}{|c|c|c|c|c|c|c|c|}
\hline Metal & $\begin{array}{c}\text { Radioisotop } \\
\text { e }\end{array}$ & $\begin{array}{c}\text { Half-life } \\
\left(t_{1 / 2}\right)\end{array}$ & $\begin{array}{l}\text { Decay } \\
\text { mode }\end{array}$ & $\begin{array}{c}\beta_{\max } \mathrm{MeV}(\% \\
\text { intensity) }\end{array}$ & $\begin{array}{l}\text { y keV (\% } \\
\text { intensity) }\end{array}$ & $\begin{array}{l}\text { Imaging } \\
\text { modality }\end{array}$ & $\begin{array}{l}\text { Production } \\
\text { method }\end{array}$ \\
\hline \multirow[b]{2}{*}{ Scandium (Sc) } & ${ }^{44} \mathrm{Sc}$ & $3.97 \mathrm{~h}$ & $\beta^{+} / E C$ & $1.474(94.27)$ & 1157 (99.9) & PET & Cyclotron \\
\hline & ${ }^{47} \mathrm{Sc}$ & $3.3492 \mathrm{~d}$ & $\beta^{-}$ & $0.600(31.6)$ & $159.38(68.3)$ & $\begin{array}{c}\text { SPECT/ } \\
\text { theranostic }\end{array}$ & Cyclotron \\
\hline \multirow{4}{*}{ Copper (Cu) } & ${ }^{61} \mathrm{Cu}$ & $3.339 \mathrm{~h}$ & $\beta^{+} / E C$ & $1.216(51)$ & $\begin{array}{l}282.96(12.2) \\
656.01(10.8) \\
\end{array}$ & PET & Cyclotron \\
\hline & ${ }^{62} \mathrm{Cu}$ & $9.67 \mathrm{~m}$ & $\beta^{+} / E C$ & $2.937(97.6)$ & - & PET & Generator \\
\hline & ${ }^{64} \mathrm{Cu}$ & $12.701 \mathrm{~h}$ & $\beta^{+} / \beta^{-} / E C$ & $0.653(17.6)$ & $511(35.2)$ & $\begin{array}{c}\text { PET/ } \\
\text { theranostic }\end{array}$ & Cyclotron \\
\hline & ${ }^{67} \mathrm{Cu}$ & $61.83 \mathrm{~h}$ & $\beta / \gamma$ & $0.377(51)$ & $\begin{array}{c}91.27(7) \\
93.31(16.1) \\
184.58(48.7)\end{array}$ & $\begin{array}{l}\text { SPECT/ } \\
\text { theranostic }\end{array}$ & Cyclotron \\
\hline Gallium (Ga) & ${ }^{66} \mathrm{Ga}$ & $9.49 \mathrm{~h}$ & $\beta^{+} / \mathrm{EC}$ & $4.153(51)$ & $833.53(5.9)$ & PET & Cyclotron \\
\hline
\end{tabular}




\begin{tabular}{|c|c|c|c|c|c|c|c|}
\hline & & & & & $\begin{array}{c}1039.22(37) \\
2751.84(22.7)\end{array}$ & & \\
\hline & ${ }^{67} \mathrm{Ga}$ & $3.262 \mathrm{~d}$ & $\mathrm{EC} / \mathrm{Y}$ & - & $\begin{array}{c}93.31(38.81) \\
184.58(21.41) \\
300.22(16.64) \\
393.53(4.56)\end{array}$ & SPECT & Cyclotron \\
\hline & ${ }^{68} \mathrm{Ga}$ & $67.71 \mathrm{~m}$ & $\beta^{+} / \mathrm{EC}$ & $1.899(87.72)$ & - & PET & Generator \\
\hline Rubidium (Rb) & ${ }^{82} \mathrm{Rb}$ & $1.258 \mathrm{~m}$ & $\beta^{+} / \mathrm{EC}$ & $\begin{array}{l}2.601(13.13) \\
3.378(81.76)\end{array}$ & 776.52 (15.08) & PET & Generator \\
\hline \multirow[b]{2}{*}{ Yttrium (Y) } & ${ }^{86} \mathrm{Y}$ & $14.74 \mathrm{~h}$ & $\beta^{+} / E C$ & $1.221(11.9)$ & $443.13(16.9)$ & PET & Cyclotron \\
\hline & ${ }^{90} \mathrm{Y}$ & $64 \mathrm{~h}$ & $\beta^{-}$ & $2.280(99.99)$ & - & $\begin{array}{c}\text { SPECT/ } \\
\text { theranostic }\end{array}$ & Generator \\
\hline \multirow[b]{2}{*}{ Zirconium (Zr) } & ${ }^{89} \mathrm{Zr}$ & $78.41 \mathrm{~h}$ & $\beta^{+} / \mathrm{EC}$ & $0.902(22.74)$ & $909.15(99.04)$ & PET & Cyclotron \\
\hline & ${ }^{97} \mathrm{Zr}$ & $16.749 \mathrm{~h}$ & $\beta / / \gamma$ & $1.916(87.8)$ & 743.36 (93.09) & $\begin{array}{l}\text { SPECT/ } \\
\text { theranostic }\end{array}$ & Reactor \\
\hline \multirow[t]{2}{*}{$\begin{array}{l}\text { Technetium } \\
\text { (Tc) }\end{array}$} & ${ }^{94 \mathrm{~m}} \mathrm{Tc}$ & $52 \mathrm{~m}$ & $\beta^{+} / E C$ & $\begin{array}{c}1.592(8.9) \\
1.938(4.1) \\
2.438(67.6) \\
3.460(12.8)\end{array}$ & $871.091(94)$ & PET & Cyclotron \\
\hline & ${ }^{99 m} \mathrm{Tc}$ & $6.01 \mathrm{~h}$ & IT & - & $140.51(89)$ & SPECT & Generator \\
\hline Indium (In) & ${ }^{111}$ In & $2.8047 \mathrm{~d}$ & $\mathrm{EC} / \mathrm{Y}$ & - & $\begin{array}{l}171.28(90.7) \\
245.35(94.1)\end{array}$ & SPECT & Cyclotron \\
\hline Lutetium (Lu) & ${ }^{177} \mathrm{Lu}$ & $6.647 d$ & $\beta / / \gamma$ & $\begin{array}{l}0.177(11.61) \\
0.4983(79.4)\end{array}$ & $\begin{array}{c}112.95(6.17) \\
208.37(10.36)\end{array}$ & $\begin{array}{c}\text { SPECT/ } \\
\text { theranostic }\end{array}$ & Reactor \\
\hline
\end{tabular}

\subsubsection{Chelator-mediated radiolabeling with radiometals}

The conjugation of radiometals to radiopharmaceuticals is typically achieved by chelation, the coordination of the radiometal cation by a chelating agent. In order to use the radiometals in biological applications, the radiometal cation must form a stable complex with the chelator to avoid undesirable hydrolysis and transchelation (the displacement of the radiometal by other metal ions in chelator cavity) in vivo. For conjugation to the targeting vector, a number of bifunctional chelating agents (BFCs) have been designed and developed. BFCs generally contain nitrogen $(\mathrm{N})$ and oxygen $(\mathrm{O})$ atoms in their structures that can donate an electron pair to form dative covalent bonds with the metal ions. Moreover, at least one side chain of the BFCs is usually modified with a functional group that can be covalently conjugated to the targeting molecules. The functional groups attached to the carbon backbone of the chelators are preferable due to the availability of all of the $\mathrm{N}$ and $\mathrm{O}$ atoms for the radiometal coordination, resulting in better stability of metal-chelator complex. A radiometal-labeled radiopharmaceutical consists of three parts: the chelating agent that forms complexes with radiometals, the spacer or linker that is covalently coupled between the chelate and the vector in order to keep the often charged radiometal complex away from the target binding motive, and the biomolecule or targeting vector, such as an antibody, peptide or nanoparticle (Figure 6).

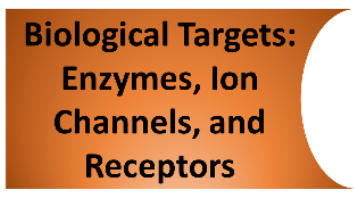

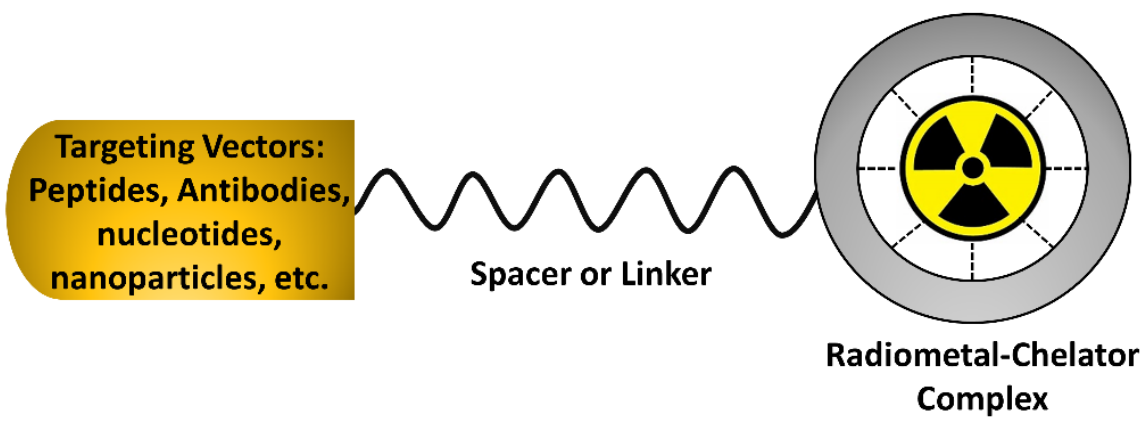

Figure 6. The general design of radiometal-labeled radiopharmaceuticals. 
The physical and chemical properties of the radiometal-chelator complex affect the overall pharmacokinetic properties of the radiolabeled compounds. Most of the radiometal-chelator complexes are very hydrophilic and have a positive charge leading to rapid clearance from the biological system through renal excretion. Therefore, the chelating agent and radiometal must be carefully matched to tailor the complex stability and charge. In general, the radiometal-chelator pair is selected based on the chelating agent and radionuclide properties, for example, ionic charge, ionic radius, cavity size in the chelator, the number of donor binding groups, and the rate of metal complex formation and dissociation. Table 2 shows currently used radiometal chelators and their bifunctional derivatives, the appropriate radiometal and typical radiolabeling conditions[6]. Since it is preferable to conjugate the chelator to the nanosystem before the radiometal complexation to avoid losses of radioactivity, the radiolabeling condition is as important as other factors mentioned previously as the construct needs to withstand the radiolabeling conditions. Room temperature and neutral $\mathrm{pH}$ conditions are superior when the labeled molecules are heat and $\mathrm{pH}$ sensitive, such as in the case of nucleotides and antibodies. Moreover, the labeling time is crucial when working with short half-life radiometals, such as scandium-44 $\left({ }^{44} \mathrm{Sc}\right)$,

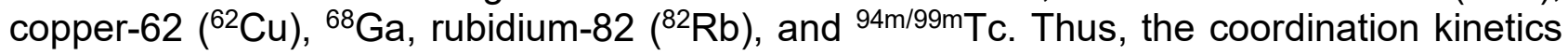
must be fast (5-15 min reaction times) and yield high radiolabeling efficiency. Macrocyclic chelators having inherently constrained geometries where the coordinating groups are close to another creating a "scaffold" metal ion binding sites undergo only minor conformational and loss in entropy upon radiometal coordination. In contrast, acyclic chelators such as desferrioxamine (DFO) must undergo a more drastic change in their geometry in order to arrange the donor atoms to coordinate with the metal ion[6]. However, despite this thermodynamic favorability of radiometal coordination to macrocycles, they generally require heating $\left(60-90^{\circ} \mathrm{C}\right)$ and longer reaction times $(>30 \mathrm{~min})$ to attain radiolabeling at a reasonable yield. In acyclic chelators the coordination often occurs at room temperature within 15 minutes. The radiometal-chelator complex stability should be validated in terms of thermodynamic stability, possibility of transchelation (e.g. Fe ${ }^{3+}$, EDTA, and DTPA), acid catalyzed dissociation, and stability in physiological conditions relevant to the administration route.

Overall, several factors need to be taken into consideration when designing chelatormediated radiolabeling of theranostic nanosystems. The choice of the radiometal is the foremost consideration as the physical half-life should be matched to the timeframe of the experiment and the nuclear properties to the theranostic application. From the standpoint of the radiometal-chelator complex the stability in vitro and in vivo, the radiolabeling conditions (media, $\mathrm{pH}$, time and temperature), and the conjugation chemistry available for nanosystem labeling all need to be taken into account.

Table 2. Common radiometal chelators, their derivatives, relevant radiometal ions, and radiolabeling conditions. Table adapted from reference [6].

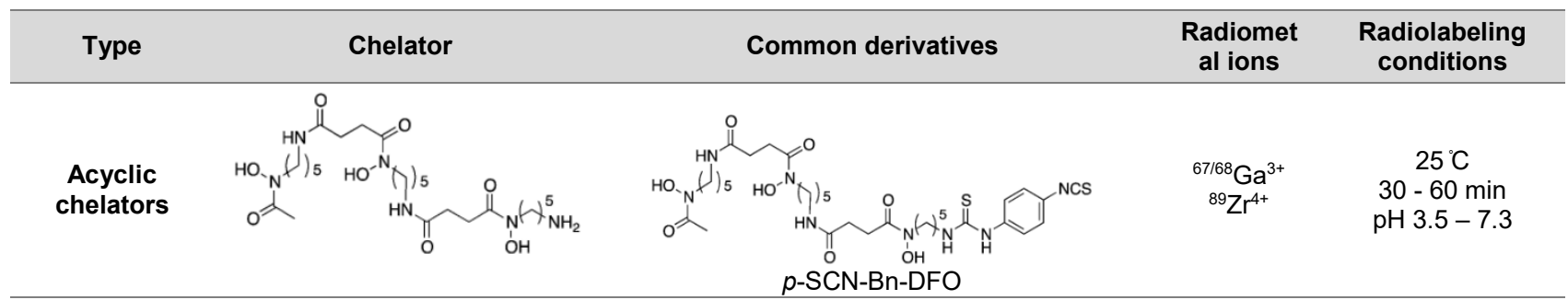


Desferrioxamine (DFO)

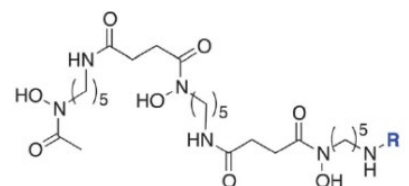

Bifunctional DFO, $\mathrm{R}=$ amide, $\mathrm{NHS}$ ester, etc.

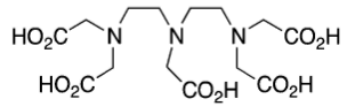

Diethylenetriaminepentaaceti $c$ acid (DTPA)

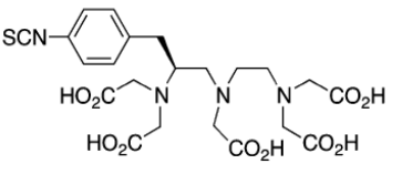

p-SCN-Bn-1B-DTPA

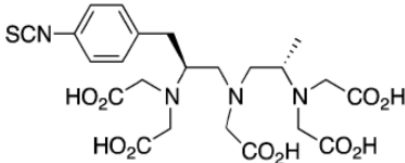
p-SCN-Bn-1B4M-DTPA<smiles>O=C(O)CN(CCN(CC(=O)O)CC1CCCCC1N(CC(=O)O)CC(=O)O)CC(=O)O</smiles>
CHX-A"'-DTPA

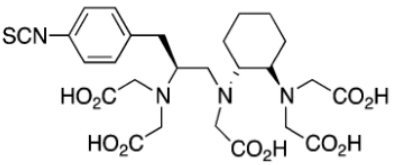
p-SCN-Bn-CHX-A"'-DTPA

Macrocyclics

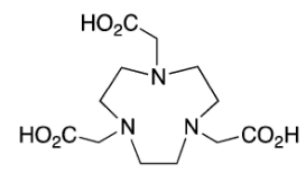

1,4,7-triazacyclononane1,4,7-triacetic acid (NOTA)

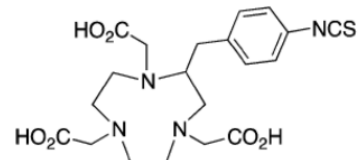

p-SCN-Bn-NOTA (C-NOTA)

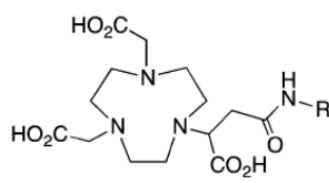

NODASA, R = NHS ester, amine, etc<smiles>[R]NC(=O)CCC(C(=O)O)N1CCN(CC(=O)O)CCN(CC(=O)O)CC1</smiles>

NODAGA, R = NHS ester, amine, etc

Table 2.2 Continued

\begin{tabular}{|c|c|c|c|c|}
\hline Type & Chelator & Common derivatives & $\begin{array}{l}\text { Radiomet } \\
\text { al ions }\end{array}$ & $\begin{array}{c}\text { Radiolabeling } \\
\text { conditions }\end{array}$ \\
\hline $\begin{array}{l}\text { Macrocyclics } \\
\text { (cont.) }\end{array}$ & 10 & DOTA-NHS-ester & $\begin{array}{c}{ }^{67 / 68} \mathrm{Ga}^{3+} \\
{ }^{44 / 47} \mathrm{Sc}^{3+} \\
{ }^{111} \mathrm{In}^{3+} \\
{ }^{177} \mathrm{Lu}^{3+} \\
{ }^{86 / 90} \mathrm{Y}^{3+} \\
{ }^{212} \mathrm{~Pb}^{2+} \\
{ }^{225} \mathrm{Ac}^{3+}\end{array}$ & $\begin{array}{c}25-9^{\circ} \mathrm{C} \\
10-120 \mathrm{~min} \\
\mathrm{pH} 4-6\end{array}$ \\
\hline
\end{tabular}


$1,4,7,10$-tetraazacyclododecane- $1,4,7,10-$
tetraacetic acid (DOTA)

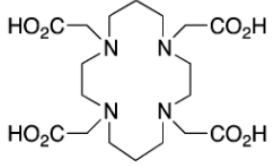

1,4,8,11-tetraazacyclotetradecane1,4,8,11-tetraacetic acid (TETA)

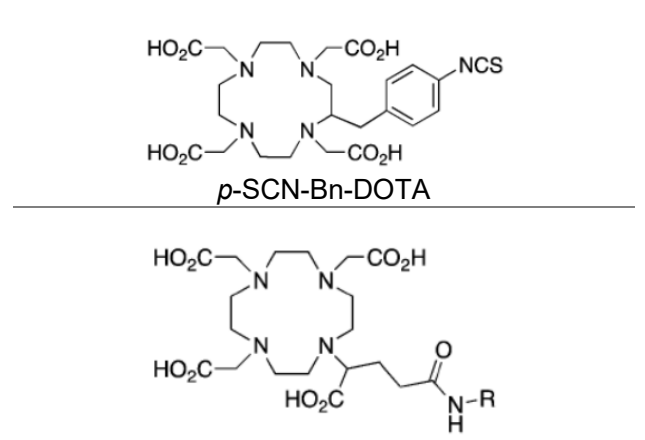

DOTAGA, $\mathrm{R}=$ amide, $\mathrm{NHS}$ ester, etc.

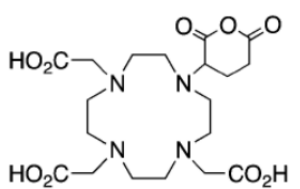

DOTAGA-anhydride

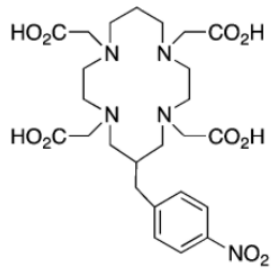

$p-\mathrm{NO}_{2}-\mathrm{Bn}-\mathrm{TETA}(\mathrm{C}-\mathrm{TETA})$

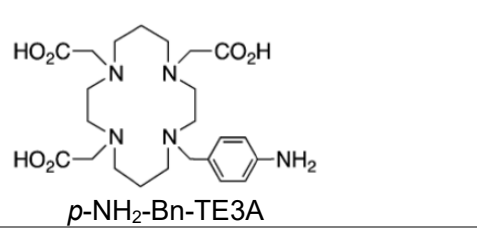

${ }^{64} \mathrm{Cu}^{2+}$

$25-95^{\circ} \mathrm{C}$

$60 \mathrm{~min}$

$\mathrm{pH} 5-7$<smiles>O=C(O)CN(CCO)CCN1CCN(CC(=O)O)CCN(CC(=O)O)CC1</smiles>

\subsubsection{Chelator-free radiolabeling}

Chelator-based radiometal labeling has been widely employed in nuclear imaging and radiotherapeutic development with radiometals. However, a number of challenges in chelator-based radiolabeling approach still remain. First, the molecules may not be able to tolerate the severe radiolabeling conditions, such as acidic $\mathrm{pH}$ and high temperature, leading to instability and destruction. Second, the incorporation of the hydrophilic chelator onto the surface of nanosystems can change their overall pharmacokinetics, surface charge, and hydrodynamic size, resulting in completely different properties from the native vector. Moreover, the coordination chemistry is vastly different between different chelators and radiometals necessitating a careful evaluation of radiometal and chelator suitability for the application. However, as radiometals can be directly coordinated to certain functional groups that either intrinsically exist in the nanoparticle structure or that are grafted on the surface of nanoparticles, chelate-free strategies have shown potential to maintain the native properties of the nanoparticles in a way that better reflects the behavior of non-radiolabeled nanoparticles in vivo. 
Several approaches to chelate-free radiolabeling of nanoparticles have been proposed to match different nanoparticle surface characteristics and modifications. Chelator-free $\left[{ }^{64} \mathrm{Cu}\right] \mathrm{CuS}$ nanoparticles has been developed as a platform for the simultaneous PET/CT imaging and photothermal therapy[7]. The $\left[{ }^{64} \mathrm{Cu}\right] \mathrm{CuS}$ can be formed by direct synthesis from the radioactive precursor in which the ${ }^{64} \mathrm{Cu}$ is incorporated into the building block of CuS and the high radiolabeling yield was achieved. A similar direct radiolabeling approach has been documented in monoclonal antibodies radiolabeling with rhenium-188 for radioimmunotherapy[8]. The protein disulfide bridges are firstly reduced with different reducing agents, generating the thiol groups ( $\mathrm{R}-\mathrm{SH})$ that can coordinate with reduced oxidation state of $\left[{ }^{188} \mathrm{Re}\right] \mathrm{ReO}_{4}^{-}$in the presence of stannous chloride $\left(\mathrm{SnCl}_{2}\right)$ as reducing agent (Figure 7).

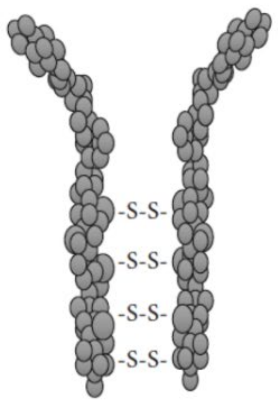

S-S- Disulfide bridges

( ${ }^{188} \mathrm{Re}$
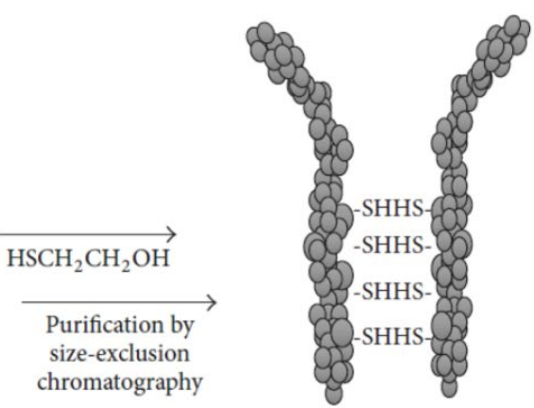

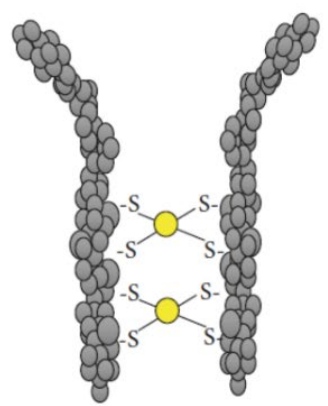

Figure 7. The direct radiolabeling approach of ${ }^{188} \mathrm{Re}$-labeled antibodies with [ ${ }^{188} \mathrm{Re}^{\mathrm{ReO}} \mathrm{Re}_{4}^{-}$using oxalate procedure. Figure reprinted with permission from reference [8].

The heat-induced radiolabeling (HIR) of radioactive cations has been employed in the radiolabeling of superparamagnetic iron oxide nanoparticles[9-11]. The thermal stability of iron oxide nanoparticles allows the high heat activation of the iron oxide core to coordinate with radiometals (e.g. ${ }^{64} \mathrm{Cu},{ }^{89} \mathrm{Zr},{ }^{111} \mathrm{In}$, and ${ }^{59} \mathrm{Fe}$ ) without changing the structure, magnetic properties and size of the nanoparticles. In addition, the selective binding of radiometals to the surface of nanoparticles has been reported. The layered double hydroxide (LDH) nanoparticles have demonstrated the potential to be readily radiolabeled with radiometals without the aid of chelators[12]. Bivalent and trivalent cations (e.g. ${ }^{64} \mathrm{Cu}^{2+}$ and ${ }^{44} \mathrm{Sc}^{3+}$ ) can form stable coordination with high labeling efficiency into the brucite-like cationic layers of the $\mathrm{LDH}$ while the tetravalent cation like ${ }^{89} \mathrm{Zr}^{4+}$ cannot efficiently label onto the LDH layer due to the lack of compatibility between the ionic radius and the LDH crystal structure. Also, a similar strategy has been applied to investigate the stereotype of chelator-free radiolabeling nanographene with ${ }^{64} \mathrm{Cu}[13]$. The reduced graphene oxide (RGO) exhibits a superior intrinsically ${ }^{64} \mathrm{Cu}$ radiolabeled to the nanographene due to the transition metal- $\pi$ electron interaction where the electron transfer takes place between ${ }^{64} \mathrm{Cu}^{2+}$ and $\pi$-bond, providing an alternative approach to maintain the properties of nanographene in vivo. The direct implantation method of theranostic radiolanthanoid, dysprosium-159 ( ${ }^{159} \mathrm{Dy}$ ) to thermally hydrocarbonized porous silicon (THCPSi) nanoparticles has been reported[14]. The THCPSi substrates are irradiated with radioactive ion beam (RIB) of ${ }^{159} \mathrm{Dy}$ at the intensity of $10^{10}$ particles/s over $39 \mathrm{~h}$, resulting in the implantation of ${ }^{159} \mathrm{Dy}$ ions on the mesoporous layer substrate. The [ $\left.{ }^{159} \mathrm{Dy}\right] \mathrm{THCPSi}$ nanoparticles demonstrated good stability 
in vivo after 7 days administration as the ${ }^{159}$ Dy remained embedded in THCPSi nanoparticles inside the tumor, prompting the use of other RIB-based radiolanthanoids for chelator-free radiolabeling with mesoporous nanoparticles. Overall, the chelator-free radiolabeling strategies reveal the potential of alternative approaches for radiolabeling with radiometals. However, understanding of the coordination mechanism between the radiometal and the nanoparticle itself as well as the stability of the radiometal complex are always warranted before in vivo applications.

\subsection{Radiohalogenation}

Radiohalogenation can be performed using typical organic chemistry techniques such as nucleophilic and electrophilic substitution. The chemistry is chosen such that new bond is strong enough to withstand physiological conditions. The carbon-halogen bond energy decreases with increase in size of halogens from $\mathrm{F}^{-}<\mathrm{Cl}^{-}<\mathrm{Br}^{-}<\mathrm{I}^{-}$. The bond strength increases with increasing number of $\pi$ bonds of the carbon. Aromatic carbon-halogens bonds have higher bond strengths compared to the aliphatic counterparts with hybridization. This makes radioiodination on aromatic substrates less prone to hydrolysis and $\beta$ elimination. Direct radioiodination technique uses electrophilic and nucleophilic substitutions as well as electrophilic addition to unsaturated compounds in certain cases.

Electron rich aromatic compounds such as those containing the activating hydroxyl, amine and methoxy functionalities can be radioiodinated using electrophilic aromatic substitution. The substitution of the hydrogen at the ortho or para positions relative to the activating groups is called iododeprotonation. The ortho and para positions relative to the electron donating group are substituted due to the higher delocalization of charge in the transition state as shown in Figure $\mathbf{8}$ for the dopamine $\mathrm{D}_{2}$ receptor radioligand, IBZM.<smiles>CCN1CCC[C@H]1CNC(=O)c1c(O)cccc1OC</smiles>

BZM

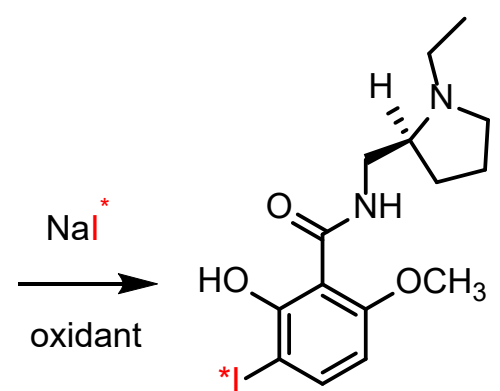

IBZM

Figure 8. lododeprotonation of BZM

For less activated aromatic rings, the synthesis is carried out in two steps with the formation of an activated derivative followed by the substitution with an electropositive radioiodine. The most commonly used organometallic precursors for iododemetallation are alkylstannyl compounds along with silyl and boronic acid derivatives. Carbon-metal bond is more activated for an electrophilic attack than a carbon-hydrogen bond. The drawback of using tri-alkyltin derivatives in the toxicity of tin compounds. Silyl derivatives are less toxic and is desirable when the radioiodinated compound is to be used for in vivo studies. The siliconcarbon bond is stronger than tin-carbon bond which results is slower reaction rates for iododesilylation. Boron derivatives also have low toxicity and boron compounds such as boronate esters and trifluoroborate salts are air stable crystalline solids making them ideal precursors for halodemetallation reactions.[15] The boronate esters and trifluoroborate salts can be regioselectively prepared using the Miyaura borylation reaction which is the coupling 
of the bis(pinacolato)diboron with aryl/vinyl halides using palladium catalyst as shown in Figure 9. Mild basic conditions for the borylation reaction is crucial to prevent activation of the borylated product and undergo Suzuki coupling reaction.[16] The ipso substitution of the metal with the iodine is regioselective under mild oxidative conditions. [ ${ }^{131}$ I]MIBG has been prepared using iododeboronation as shown in Figure 10.

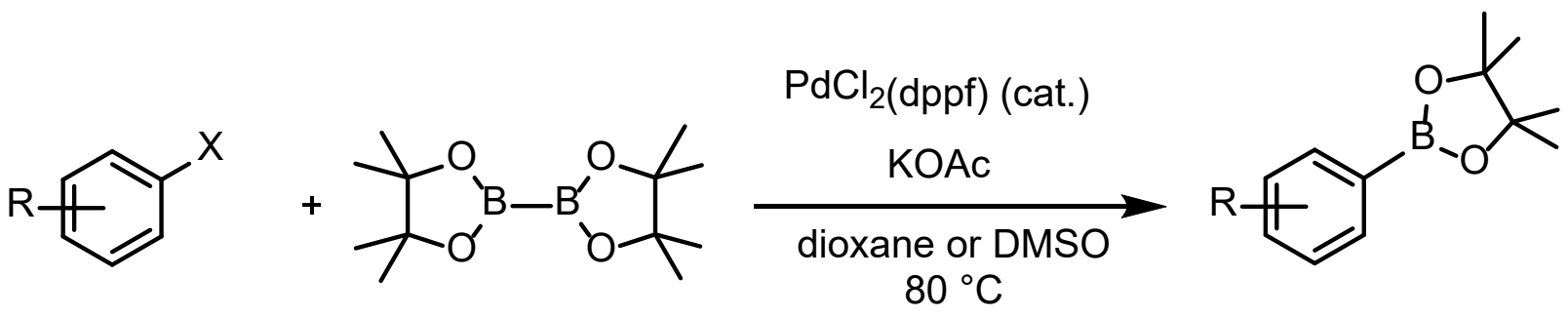

$\mathrm{X}=\mathrm{Br}, \mathrm{I}, \mathrm{OTf}$

Figure 9. Miyaura borylation reaction<smiles>N=C(N)NCc1cccc(B(O)O)c1</smiles><smiles>N=C(N)NCc1cccc(I)c1</smiles>

Dimethyl carbonate

$\mathrm{RCY}=91 \%$

(solvent)
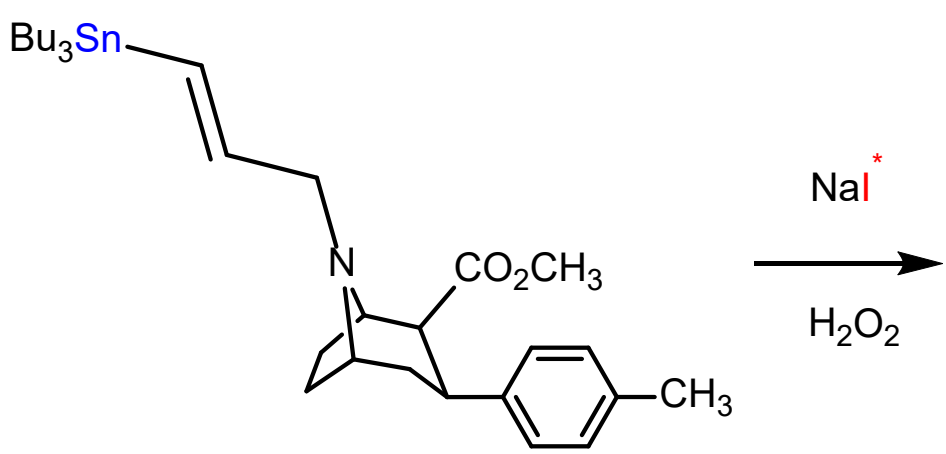

Figure 10. Synthesis of [ $\left.{ }^{131} \mathrm{I}\right] \mathrm{MIBG}$ using iododeboronation (top) and PE2I using iododestannylation (bottom).

The radioiodine is generally obtained as sodium iodide. It has to be oxidized to its unipositive charged state for electrophilic reactions. It can be oxidized using water soluble oxidants such as chloramine-T, N-chlorosuccimide (NCS) and peracids [17]. Chloramine-T is a strong oxidant but maybe lead to by-products. Low reaction temperatures and fresh reagents are key to successful oxidation. Polymer bound chloramine called lodobeads [18] has been developed to reduce exposure of oxidant sensitive molecules. lodogen is another chlorinebased oxidant but it is milder and hence can be used with proteins and peptides. lodogen is water insoluble and it typically used as a thin film on a glass vial. lodogen is compatible with common detergents which makes is widely applicable for labeling biologics. Radiolabeled proteins have been shown to have different stabilities depending on the source of the 
oxidizing agent showing there are fundamental differences among the mechanisms of radioiodination.[19] Structure of some of the oxidants are shown in Figure 11.<smiles>O=S(=O)([O-])c1ccccc1</smiles>

Chloramine-B<smiles>Cc1ccc(S(=O)(=O)N([O-])Cl)cc1</smiles>

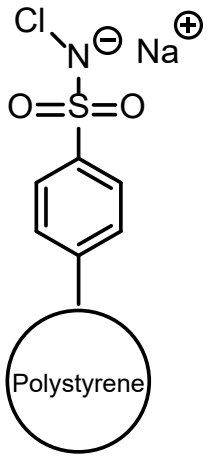

lodobeads<smiles>O=C1N(Cl)C2(c3ccccc3)N(Cl)C(=O)N(Cl)C2(c2ccccc2)N1Cl</smiles>

lodogen $^{\mathrm{TM}}$

Figure 11. Structure of some chlorine-based oxidants

Radioiodination conditions are harsh for sensitive molecules such as peptides and antibodies. To prevent such exposure, indirect radiolabeling methods are used in which prosthetic groups such as Bolton-Hunter reagent are radioiodinated, which can then be conjugated to biomolecules.[20]. A typical conjugation of a radioiodinated Bolton-Hunter reagent is shown in Figure 12. Other spacers which are bifunctional and can be used for radioiodination are shown in Figure 13. These allow versatile bioconjugation to sulfhydryl and amine containing compounds.
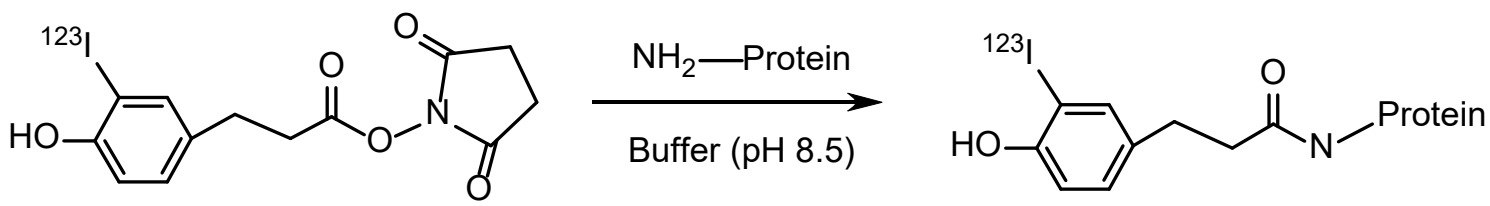

Figure 12. Conjugation of a protein to a radioiodinated Bolton-Hunter reagent

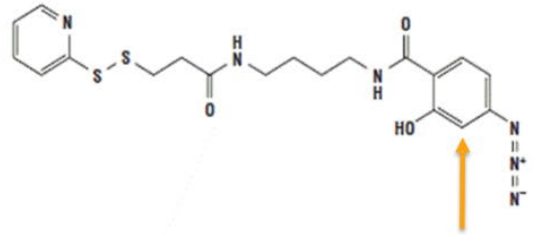

APDP<smiles>CCCCNC(C)C1CCC(CC)C(C)C1C</smiles>

ASBA

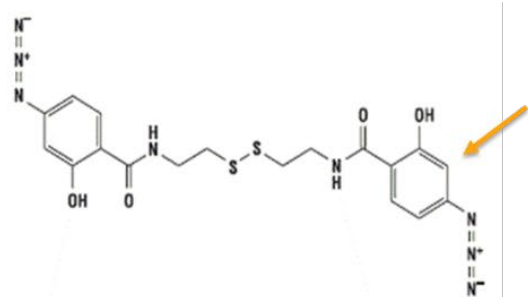

BASED

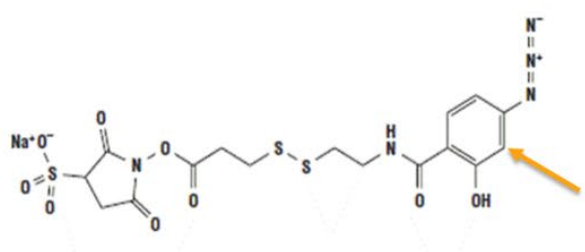

SASD

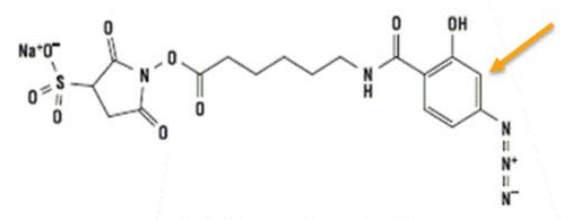

Sulpho-NHS-LC-ASA

Figure 13. Spacers which can be radioiodinated; radioiodination positions have been marked. 
Nucleophilic substitution is carried out in compounds which are functionalized with electronwithdrawing groups where the carbon is functionalized with good leaving groups such as meylate, triflate, tosylate or brosylate as shown in Figure 14.<smiles>CS(=O)(=O)[O-]</smiles>

Mesylate<smiles>O=S(=O)([O-])C(F)(F)F</smiles>

Triflate<smiles>Cc1ccc(S(=O)(=O)[O-])cc1</smiles>

Tosylate<smiles>O=S(=O)([O-])c1ccc(Br)cc1</smiles>

Brosylate

Figure 14. Structure of leaving groups used in nucleophilic substitution reactions

Aliphatic carbons can use either $\mathrm{S}_{\mathrm{N}} 1$ or $\mathrm{S}_{\mathrm{N}} 2$ mechanisms and aromatic compounds use $\mathrm{S}_{N} \mathrm{Ar}$ mechanism. The rate-determining step for a $\mathrm{S}_{N} A r$ reaction is the attack of the nucleophile and formation of the intermediate complex. For increasing the reaction rate, the labeling position needs to be activated by electron withdrawing groups such as $-\mathrm{NO}_{2}$, aldehyde, ketone, ester, amide in ortho- or para-position or catalyzed by metal salts such as Cu-salts.

For aryl amines the classic Sandmeyer-type reaction can be performed by diazotization of the amine, followed by copper(I) catalyzed radioiodination. Limitations of such a reaction are harsh acidic conditions required for the formation of the diazonium salt and the potentially explosive diazonium salts. A one-pot radioiodination was reported where the diazonium salts were formed under mild reaction conditions. This was achieved using a polymer-supported nitrite reagent and p-toluene-sulphonic acid as a proton source, followed by the direct radioiodination. The solid phase support allows for simple and rapid purification. [21]

Substitution of an iodine substituent with radioiodine is called homo/isotopic exchange whereas that of a bromine substituent is called hetero/halogen exchange. These reactions are typically referred to as radioiododehalogenation. Even though commonly used, the isotopic exchange suffers from low molar activity since the parent molecule cannot be separated from the product. When a bromine is being substituted with radioiodine the product can often be separated using chromatographic methods leading to higher specific activity. Ammonium sulphate facilitates solid-phase exchange radioiodination of unactivated aryl iodide. The process involves heating to partial decomposition of the salt to lower $\mathrm{pH}$ from 6 to 3 which is essential for the reaction as shown in Figure 15.<smiles>N=C(N)NCc1cccc(I)c1</smiles>

$\left[{ }^{125} \mathrm{I}\right](\mathrm{m}$-iodobenzyl)guanidine

Figure 15. Nucleophilic exchange reaction for the synthesis of $\left.\left[{ }^{125}\right]\right](m$-iodobenzyl)guanidine

Copper(I) catalyzes nucleophilic reactions by forming a 3-membered intermediate complex. Typically the $\mathrm{Cu}(\mathrm{I})$ is produced in situ by the reduction of $\mathrm{Cu}(\mathrm{II})$ by stannous chloride. The 
$\mathrm{pH}$ is maintained acidic to prevent nucleophilic attack by hydroxyl anions. The nucleophilic reaction can be also performed on boronated compounds as shown in Figure 16. Higher radiochemical yields were obtained than electrophilic iododeborylation for compounds containing both electron-donating and electon-withdrawing substituents.<smiles>CC(C)(C)OC(=O)N(Cc1cccc(B(O)O)c1)C(N)=[N+]([O-])O</smiles>

1. $\left[{ }^{131} \mathrm{I}\right] \mathrm{Nal}, \mathrm{Cu}_{2} \mathrm{O}, 1,10-$ Phenanthroline,

2. Trifluoroacetic acid<smiles>N=C(N)NCc1cccc(Br)c1</smiles>

Figure 16. Copper(I) catalyzed nucleophilic radioiodination of boronated compounds.

\subsection{Radiolabeling with positron-emitting radionuclides}

The most important positron emitters for radiolabeling of therapeutic nanosystems for diagnostics purposes are ${ }^{18} \mathrm{~F},{ }^{124} \mathrm{I},{ }^{64} \mathrm{Cu}$ and ${ }^{89} \mathrm{Zr}$. All these radionuclides have sufficiently long half-lives for the radiolabeling of theranostic nanosystems, which typically have high molecular mass and slow pharmacokinetics (Table 3). From these nuclides, ${ }^{18} \mathrm{~F}$ has the most optimal imaging characteristics, but also the shortest half-life ( $\left.\mathrm{t}_{1 / 2}=109.7 \mathrm{~min}\right)$. Therefore it can be used for tracing nanosystems with faster pharmacokinetics or by adapting a pretargeted radiolabeling strategy. ${ }^{18} \mathrm{~F}$ does not have any theranostic nuclide pair, but nevertheless is an excellent choice as a diagnostic radionuclide when tracing therapeutic drug delivery systems for example for cancer chemotherapy. ${ }^{124} \mathrm{I},{ }^{64} \mathrm{Cu}$ and ${ }^{89} \mathrm{Zr}$ have their theranostic pairs as ${ }^{131} \mathrm{I},{ }^{67} \mathrm{Cu}$ and ${ }^{90} \mathrm{Y}$, which are all $\beta$-emitters and widely used as radiotherapeutic agents in anticancer therapies.

Table 3. Positron emitting diagnostic radionuclides for theranostic systems.

\begin{tabular}{ccccc}
\hline Radionuclide & Decay mode & $\begin{array}{c}\text { Half-life } \\
\left(\boldsymbol{t}_{1 / 2}\right)\end{array}$ & $\begin{array}{c}\mathrm{E}_{\text {mean }} \boldsymbol{\beta}^{+} \\
\mathbf{M e V}(\% \\
\text { intensity) }\end{array}$ & Theranostic pair \\
\hline${ }^{18} \mathrm{~F}$ & $\beta^{+}$ & $109.7 \mathrm{~m}$ & $0.250(98)$ & - \\
\hline${ }^{64} \mathrm{Cu}$ & $\beta^{+}, \beta^{-}$ & $12.7 \mathrm{~h}$ & $0.278(9)$ & ${ }^{67} \mathrm{Cu}$ \\
\hline${ }^{89} \mathrm{Zr}$ & $\beta^{+}$ & $78.4 \mathrm{~h}$ & $0.395(13)$ & ${ }^{90} \mathrm{Y}$ \\
\hline${ }^{124} \mathrm{I}$ & $\beta^{+}$ & $4.18 \mathrm{~d}$ & $0.687(12)$ & ${ }^{131} \mathrm{I}$ \\
\hline
\end{tabular}

The stability of the radiolabeling position is of utmost importance for both the diagnostic and therapeutic use of nanosystems. Detached radionuclide may hamper the diagnostic use of a theranostic system, as the nuclear imaging will not be able to make any difference between different radioactive species. Especially if the detached radionuclide is bound to plasma proteins it may be difficult to differentiate whether the signal in circulation or tumor is originating from the protein-bound free radionuclide or from the radiolabeled theranostic system itself. For radiotherapeutic use the radiolabel instability is even more serious because the detached therapeutic radioisotope may cause serious adverse effects in radiation-sensitive healthy tissues such as the bone marrow, kidneys and the intestinal epithelium. When using a true theranostic nuclide pair with different isoptopes of the same 
element, the diagnostic and therapeutic nuclides exhibit identical chemical and pharmacokinetic properties and therefore the diagnostic system is perfectly suited for reliable evaluation of stability and biodistribution of the therapeutic system. In contrast, when radionuclides with different chemical properties are used, such in case of ${ }^{89} \mathrm{Zr} /{ }^{90} \mathrm{Y}$, more careful validation of the diagnostic accuracy of the system needs to be carried out before therapeutic use.

In principle, the chemical toolbox available for the radiolabeling of nanosystems is the same as in synthetic chemistry with stable isotopes. The only limitations for the use of short living radionuclides are the time available for the radiosynthesis and characterization, and the limited selection of the radioactive starting materials depending on production method of the selected radioisotope. The radioisotope production determines the chemical species of the produced radioisotope and the solvent or carrier gas in which they are delivered.<smiles>COC1O[C@@H](C[18F])[C@@H]2OC(C)(C)O[C@H]12</smiles>

Aromatic $\left[{ }^{18}\right.$ F]fluorination<smiles>CCOC(=O)c1ncn2c1CN(C)C(=O)c1cc([N+](=O)[O-])ccc1-2</smiles>

Transition metal mediated $\left[{ }^{18}\right.$ F]fluorination<smiles>CCOC(=O)C(Cc1cc(OC)c(OC)cc1OC)Cc1cc(OC)c(OC)cc1B1OC(C)(C)C(C)(C)O1</smiles>

Figure 17. Typical reaction conditions for $\left[{ }^{18} \mathrm{~F}\right]$ fluorination.

Fluorine can form stable covalent bonds with carbon and certain heteroatoms, such as silicon and boron. The formation of the carbon- $\left[{ }^{18} \mathrm{~F}\right]$ fluorine bond is the most difficult to attain and anhydrous reaction conditions and high temperatures are needed in order to achieve high radiochemical yields, even with reactions mediated by transition metals (Figure 17). In all cases, the stability of the bond is influenced by the groups adjacent to the $\left[{ }^{18} \mathrm{~F}\right]$ fluorine. As fluorine is the most electronegative element in the periodic table, it forms bonds which are strong, but highly polarized and can therefore be hydrolyzed by a nucleophilic attack. This can be overcome if the fluorine is stabilized by functional groups that can inductively compensate the positive partial charge of the fluorine binding atom 
and/or can sterically hinder it against the attack (Figure 18). The most stable carbon$\left[{ }^{18} \mathrm{~F}\right]$ fluorine bonds are between fluorine and an aromatic $\mathrm{sp}^{2}$-carbon, in which the delocalization of the partial positive charge to the aromatic ring can compensate for the bond polarization and decrease vulnerability of the bond against the nucleophilic attack. When fluorine is bound to a $\mathrm{sp}^{3}$-carbon, there is an additional mechanism which can lead to defluorination of the compound; in favorable conditions $\left[{ }^{18} \mathrm{~F}\right]$ fluorine can be eliminated together with an adjacent proton (Figure 19). Silicon is one of the least electronegative compounds and predominantly forms bonds as $\mathrm{sp}^{3}$-hydridized silicon. Polarization of the fluorine bond is most prominent for silicon and careful structure optimization is required in order to achieve stable $\left[{ }^{18} \mathrm{~F}\right]$ fluorination to silicon. In addition to carbon, silicon and boron, fluorine can form stable coordination complexes together with aluminum by generating an $\left[{ }^{18} \mathrm{~F}_{\mathrm{AlF}}{ }^{2+}\right.$ complex, which can be chelated analogously to several radiometals.

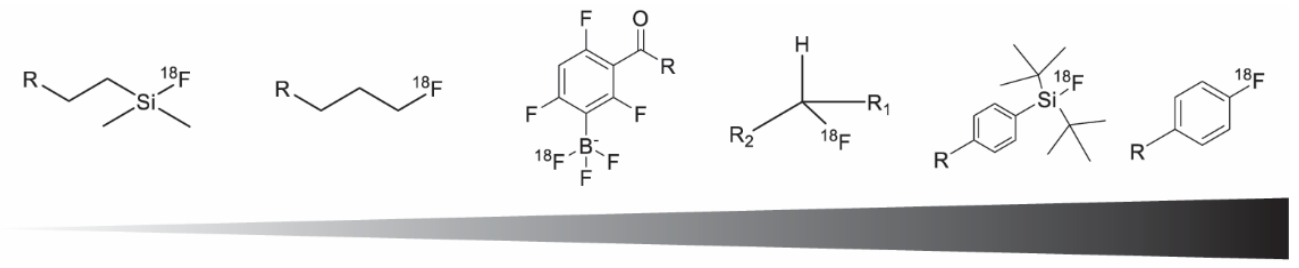

Increasing stability

Figure 18. The bond type and the position influences stability against defluorination

The radiolabelling methods for iodine-124 are described in section 3.2. Only the radioiodination to $\mathrm{sp}^{2}$-carbon is stable in vivo. Radioiodination to a saturated position leads to quick elimination of the radiolabel and accumulation of the free iodine into the thyroid. The detachment of an iodine label can never be completely avoided, but accumulation into thyroid can be minimized by pretreating the thyroid with iodine salts. Copper-64 and zirconium-89 are radiometals and can be incorporated to the nanosystems by chelation chemistry. As described already in section 3.1., i) copper-64 exists predominately in oxidation stage II and its coordination number varies between 4 and 6 . When using copper64 for radiolabelling of nanotheranostics systems, it is especially important to notice its susceptibility to bind to endogenous proteins, such as ceruloplasmin and superoxide dismutase. The protein binding competes with binding to a chelator and may cause transchelation of the radiolabel from the nanostructure to the protein. Zirconium-89 is an emerging radionuclide in radiolabeling of nanosystems, due to its long half-life and low positron energy. The main challenge with zirconium-89 is stable chelation - all the existing chelators for the four-valent ${ }^{89} \mathrm{Zr}$ have have exhibited limited in vivo stability leading to bone accumulation of detached radiometal over time.

\subsection{Pretargeted radiolabeling strategies}

Instead of using direct labeling of nanosystems prior their administration it is possible to utilize pretargeted radiolabeling strategies. In a pretargeted strategy, nanosystems are radiolabeled in vivo by using radiotracers which specifically bind to the administered nanosystem either by weak interactions or covalent bonds. One of the most investigated strategies for pretargeted radiolabeling is the biotin-(strept)avidin system, in which the recognition is based on the high-affinity interaction between biotin and avidin[22]. Other investigated pretargeted systems are for example systems based on hapten-antibody and 
DNA-DNA interactions[23, 24]. Recently, pretargeted strategies based on bioorthogonal chemical reactions have been under intensive investigation.

In bioorthogonal chemistry -based pretargeted strategies, the recognition between the tracer and a nanosystem is based on a chemical reaction, which leads to the formation of a covalent bond. Several reactions have been investigated for the purpose such as Staudinger ligation, strain-promoted cycloadditions and inverse electron-demand DielsAlder cycloaddition (IEDDA). From these only IEDDA has been shown to exhibit fast enough kinetics in physiological conditions and in living animals. In the IEDDA reaction, a cycloaddition product is formed between two functional groups, namely a tetrazine and a strained alkene such as trans-cyclooctene (TCO) (Figure 19). Typically, a radiolabeled tetrazine is used for radiolabeling of a TCO-conjugated nanosystem due to the better metabolic stability of functionalized tetrazines. Pretargeted PET imaging based on biorthogonal chemical reactions has demonstrated great potential in nanotheranostic applications and may allow for the use of short-living radionuclides for tracing longcirculating nanosystems without unnecessary radiation exposure to the patient.

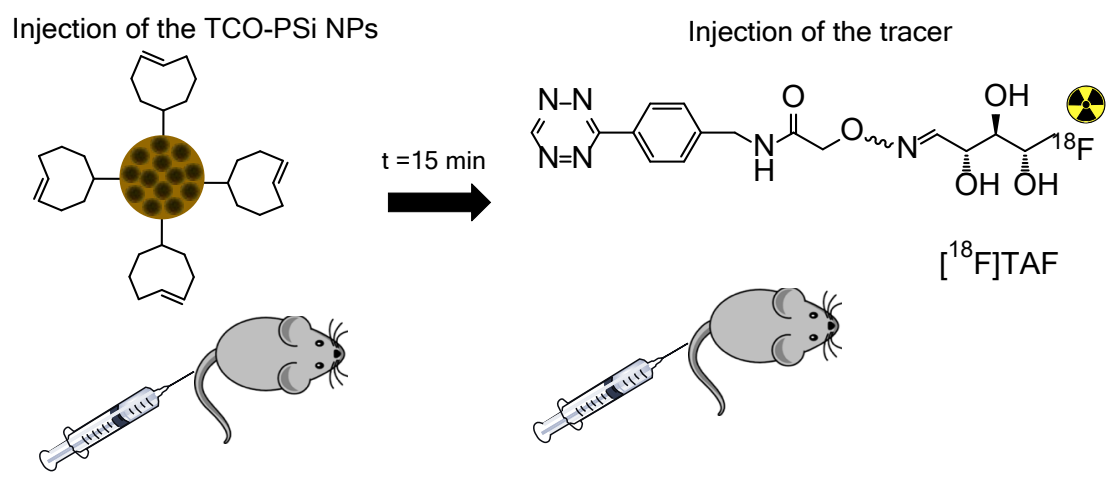

PET imaging of the in vivo radiolabeled NPs

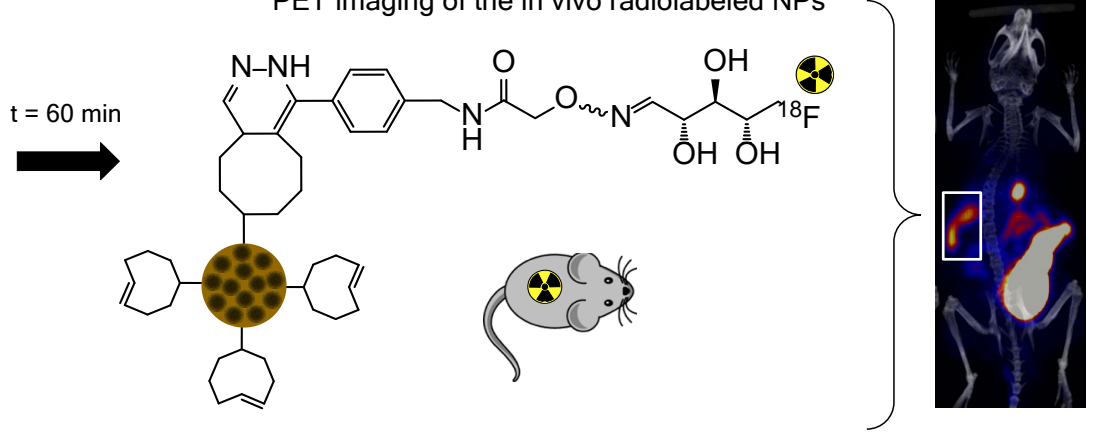

Figure 19. Pretargeted PET imaging of a TCO-conjugated porous silicon nanosystem (TCO-PSi) in mice. The TCO-PSis were injected 15 minutes before injection of a ${ }^{18} \mathrm{~F}$-labelled tracer, $\left[{ }^{18} \mathrm{~F}\right] \mathrm{TAF}$. PET imaging was performed 60 minutes after injection of the tracer and the TCO-PSis were successfully traced in spleen (delineated by a box). Some accumulation also in liver was observed. The observed high levels of radioactivity in gall bladder, intestines and urinary bladder are caused by elimination of $\left[{ }^{18} \mathrm{~F}\right] \mathrm{TAF}$ and its radioactive metabolites. Figure reprinted with permission from reference [25].

\subsection{Radiolabeling with alpha emitters}

Alpha emitters can be chelated to traditional chelators like DOTA but they are not because the recoil energy of the ${ }^{225} \mathrm{Ac}$ daughters after an $\alpha$ decay will break any metal-ligand bond. Radium-223 $\left({ }^{223} \mathrm{Ra}\right)$, lead-211 $\left({ }^{211} \mathrm{~Pb}\right)$, francium-221 $\left({ }^{221} \mathrm{Fr}\right)$ and bismuth-211 $\left({ }^{211} \mathrm{Bi}\right)$ are the primary daughter isotopes and cause renal toxicity. ${ }^{225} \mathrm{Ac}$ and its daughter ions are oxophilic 
making it easy to bind to hydroxyl right surfaces such as that of titanium dioxide. To prevent the leaching of ions due to the alpha decay they have to be encapsulated in nano-carriers. $\mathrm{TiO}_{2}$ nanoparticles were functionalized with peptide fragments targeted to glioma cells with PEG spacer. ${ }^{225} \mathrm{Ac}$ was incorporated by ion exchange on the hydroxyl groups. After 10 days of incubation in physiological salts, $95 \%$ of ${ }^{225} \mathrm{Ac}$ and ${ }^{221} \mathrm{Fr}$ was retained in the nanoparticle core.[26] This type of exchange reactions can also be performed on nanozeolites. Although produced chemically, nanozeolites have been shown to have similar toxicity to silicon nanoparticles. ${ }^{223}$ Ra labeled nanozeolites showed $90 \%$ retention after 6 days with upto $5 \%$ release of ${ }^{211} \mathrm{~Pb}$ and ${ }^{211} \mathrm{Bi}$.[27] Medically relevant poly(butadiene-b-ethylene oxide) was used to make liposomal vesicles. ${ }^{225} \mathrm{Ac}$ was chelated with ionophores like calimycin and then incorporated into the liposomes. Recoiled ${ }^{213} \mathrm{Bi}$ and ${ }^{221} \mathrm{Fr}$ could be separated from the unbound liposomes using DOWEX ion exchange resin and was found to be $47 \%$ and $31 \%$ respectively.[28]

Astatine-211 $\left({ }^{211} \mathrm{At}\right)$ is another promising alpha emitter belonging to the halogen series. As before, prosthetic groups such as succinimidyl astatobenzoate (SAB) can be used to functionalize biomolecules. SAB is synthesized from a stannyl precursor using electrophilic halodemetallation reaction with an oxidant as shown in Figure 20. The limitation of using oxidizing conditions is the multiple oxidation states available for astatine, which leads to low reproducibility and hence, limiting clinical application. With this respect, At is much easier to stabilize and hence nucleophilic reaction conditions are preferable. Nucleophilic substitution of aryliodonium salt containing prosthetic groups using At $^{-}$(Figure 21) was shown to have high radiochemical yields. The aryl group on the aryliodonium salt which should not be radioastatinated was chosen to be electron rich to prevent attack of $\mathrm{At}^{-}$ . The $p$-anisyl group achieved the best radiochemical yields for radioastatination among $p$ isopropoxyphenyl and 2-thienyl groups.[29]<smiles></smiles>

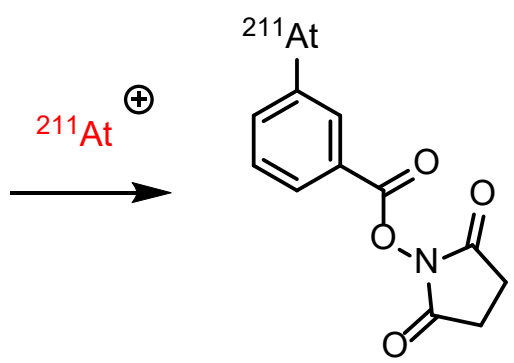

Succinimidyl astatobenzoate (SAB)

Figure 20. Synthesis of prosthetic group containing Astatine-211.<smiles>O=C(ON1C(=O)CCC1=O)c1cccc(I)c1</smiles><smiles>CC(CCC(=O)O)C(=O)Br</smiles><smiles>O=C(ON1C(=O)CCC1=O)c1cccc(I([18O])[18O])c1</smiles> 


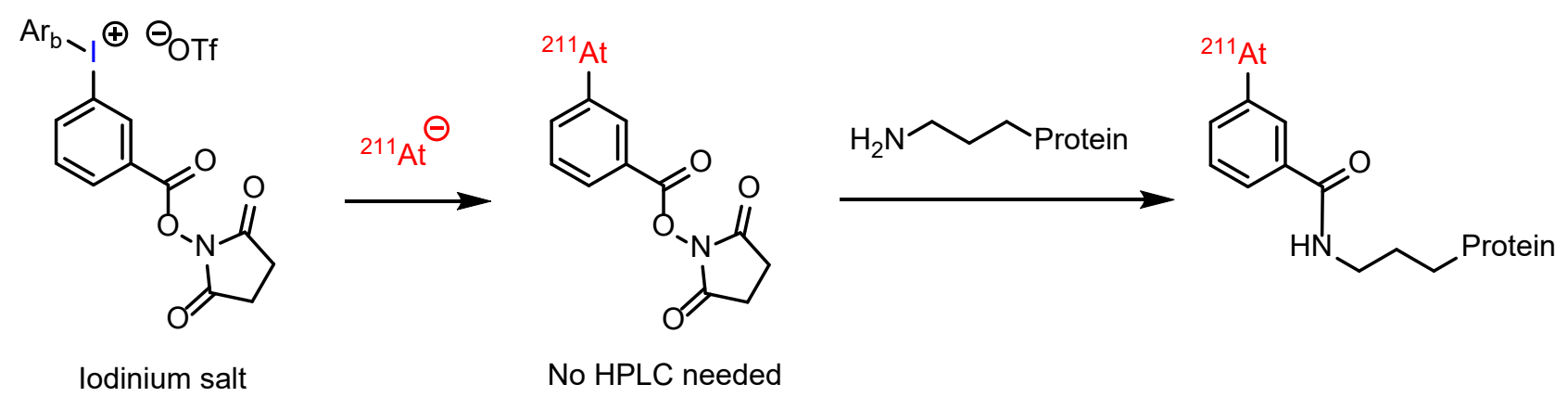

Figure 21. Using iodinium salts for nucleophilic substitution reactions.

\section{Preclinical studies with radiolabeled tracers}

Here, we give an overview of the standard preclinical in vitro and ex vivo/in vivo methods employed in the biological evaluation of radiolabeled tracers including theranostic nanosystems. The focus is on methods where the output will be a measurement of the amount of radioactivity or visualization of its biodistribution. Conventional therapeutic efficacy and survival studies are to be used alongside as for non-radioactive theranostic nanosystems, these are expertly reviewed in other chapters of this book.

\subsection{In vitro methods}

The in vitro methods used in the evaluation of theranostic nanosystems include various radiolabel stability assays in physiologically relevant media and cell uptake studies for the determination of the nanosystem cellular interaction and internalization and to corroborate the specificity of the nanosystem targeting. Additionally, as a proof-of-concept, the therapeutic effects of theranostic nanosystems can be assessed in vitro using various cell viability assays, assays for reactive oxygen species (ROS) and inflammatory markers, as well as by immunofluorescence staining of cells for markers of radiation-induced DNA damage, apoptosis, senescence and by observing the radiation-induced synchronization of the cell cycle with flow cytometry[30].

\subsubsection{Radiolabel stability assays}

Since the chemical identity of a radioactive species cannot be readily identified from nuclear images or radioactivity measurements of excised tissues - in these the radioactive signal is related only to the radioisotope but not its chemical form - radiolabel stability needs to be rigorously evaluated in vitro before proceeding to in vivo studies. Typically, the radiolabeled tracer is incubated in physiological media relevant for the administration route (plasma, serum, simulated gastric or intestinal fluids, simulated lung fluid, or cell culture media) at $37^{\circ} \mathrm{C}$ over a period of time and samples are drawn from the incubations at designated time points and analyzed for the fraction of radioactivity remaining in the intact radiotracer. In the case of nanosystems, the nanoparticles are usually removed from the incubation by centrifugation or ultrafiltration and the amount of radioactivity remaining in the collected nanoparticles and in the solution is measured. Alternatively, analytical methods such as size-exclusion chromatography (SEC) thin layer chromatography (TLC) with radioactivity detection can be used to more closely monitor for the presence of the intact radiolabeled nanoparticles and the free radiolabel. In this case, the analytical method needs to be set up so that the retention times for the two are sufficiently different and that a clear separation is obtained. Additionally, the level of radioactivity at the sample needs to be set up to a level that can provide a clear signal even after long incubations. The radiolabel stability should 
be followed for a duration of time meaningful for the in vivo application and for as long as reasonably permitted by the half-life of the radioisotope. If the theranostic nanosystem is intended for radiotherapy for example, the stability should be followed for days, for an imaging study with a shorter-lived radioisotope a duration of few hours is often sufficient. The radiolabel stability should be exceeding $95 \%$ for the entire duration the stability assay.

\subsubsection{Cell uptake and internalization assays}

Radioactivity is a convenient tool also for the quantification of the cellular internalization and targeting of theranostic nanosystems. In these assays, the radiolabeled nanoparticles are incubated with the targeted tumor cell line, and at designated time points the amount of radioactivity remaining in the media, on cell surface and in the cell lysate is determined by radioactivity measurement of the fractions. The specificity of the cellular uptake can be studied by preventing the targeting using a large molar excess of a competing ligand or nanosystems modified with for example non-targeting control sequences. The time frame for the experiment depends on the system under study, but typically studies over a few hours or even 1-2 doubling times of the cell line can be done depending on the half-life of the radioisotope and its cytotoxicity.

\subsubsection{Markers for radiation-induced cellular damage}

lonizing radiation induces a number of characteristic alterations in mammalian cells, and all of these can be used to determine the radiotherapeutic potential of the theranostic nanosystem. The DNA double-strand breaks induced by high LET $\alpha$ and $\beta^{-}$radiation can be visualized using $\gamma \mathrm{H} 2 \mathrm{AX}$, a marker of histone phosphorylation which occurs in response to the double-strand break.[31] $\gamma \mathrm{H} 2 \mathrm{AX}$ has been shown to be a robust marker for radiation damage which is illustrated by the example in Figure 22. The example from Graf and coworkers nicely illustrates by the number of $\gamma \mathrm{H} 2 \mathrm{AX}$ foci in rat pancreatic adenocarcinoma cells the differences between the LET values for theranostic $\beta^{-}$emitter ${ }^{177}{ }^{17 u}$ and the $\alpha$ emitter ${ }^{225} \mathrm{Ac}$ when delivered by the same vector, the somatostatin receptor targeting peptide DOTATOC[32]. 
A
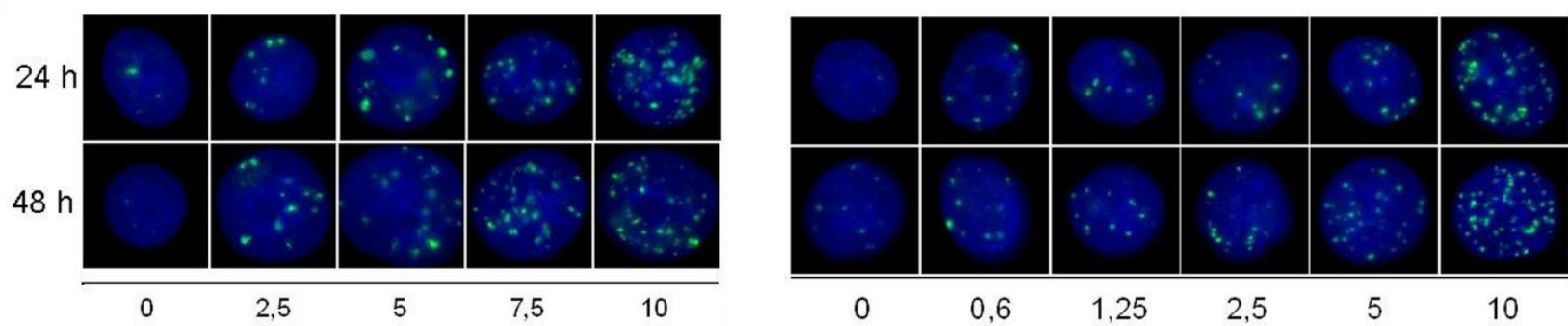

B
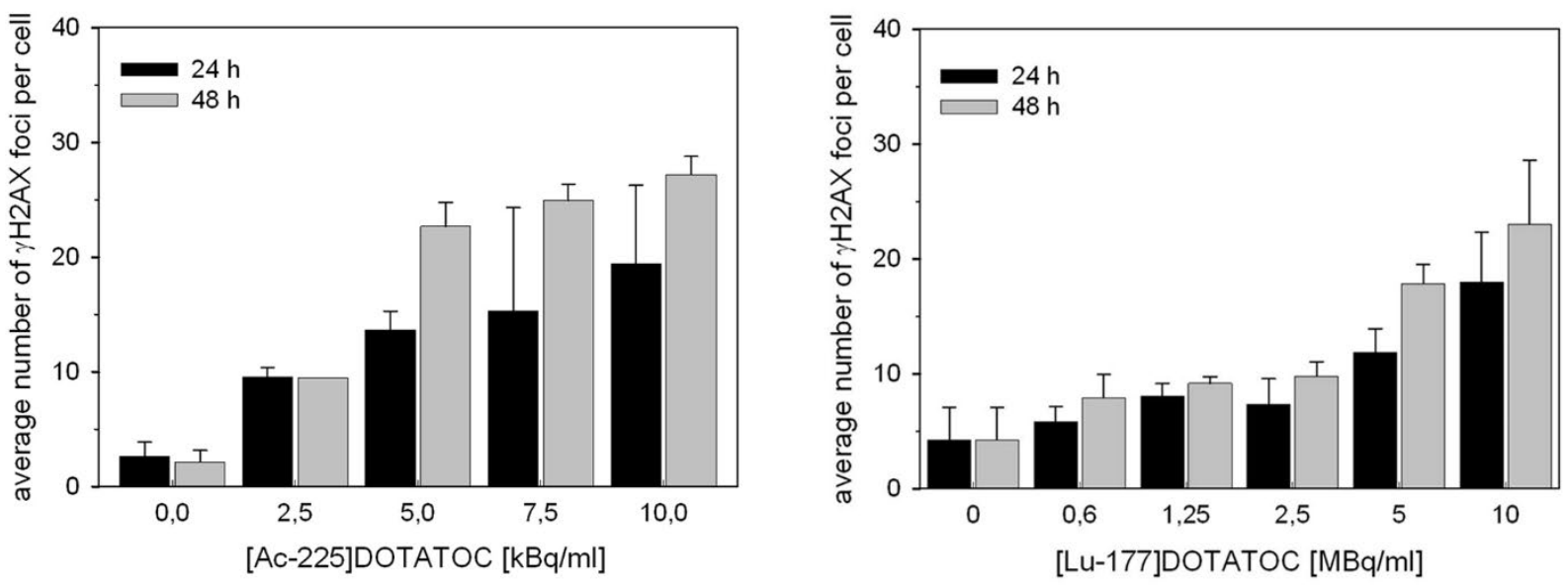

C

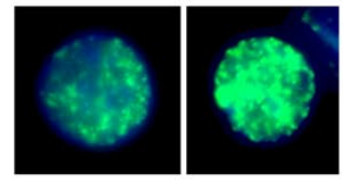

Figure 22. Histone phosphorylation marker $\gamma \mathrm{H} 2 \mathrm{AX}$ can be used to quantify the number of DNA double-strand breaks induced by radiation therapy. Number of $\gamma \mathrm{H} 2 \mathrm{AX}$ foci in AR42J cells at 24 and $48 \mathrm{~h}$ after incubation with ${ }^{225} \mathrm{Ac}$-DOTATOC (left) and ${ }^{177} \mathrm{Lu}$-DOTATOC (right). (A) shows representative images from all activity levels, (B) shows quantification of $\mathrm{cH} 2 \mathrm{AX}$ foci and (C) shows two representative examples for pan nuclear staining after high dose ${ }^{225} \mathrm{Ac}$-DOTATOC treatment. Figure reprinted with permission from reference [32].

Cells respond to the DNA damage elicited by ionizing radiation by the activation of two main pathways, one leading to apoptosis[33] and the other to premature ageing, or senescence[34]. Staining for apoptosis using established markers such as TUNEL (terminal deoxynucleotidyl transferase dUTP nick-end labeling), active caspase-3 or Annexin $V$ is widely used also in the context of radiotherapy to study cellular and tissue-level effects of radiation treatment. Entry into the premature senescent or senescence-associated secretory phenotypes (SASPs) can be studied using a number of hallmark markers for senescence, including senescence-associated $\beta$-galactosidase, decreased Ki67, and elevated trimethylated histone $\mathrm{H} 3$ lysine 9 (H3K9me3)[35, 36]. The DNA damage resulting from exposure to ionizing radiation activates checkpoint pathways that inhibit the progression of the cells through the $\mathrm{G}_{1}$ and $\mathrm{G}_{2}$ phases of the cell cycle and induce a transient delay in the progression through $S$ phase. As a result, an arrest of cells in the $\mathrm{G}_{2} / \mathrm{M}$ checkpoint can be seen. This can be studied for example by flow cytometry [30, 37].

\subsection{Ex vivo biodistribution studies and autoradiography}

The distribution of radiolabeled tracers in the body is in the preclinical setting most commonly determined in an ex vivo biodistribution study, where groups of animals $(n=3-5)$ 
are sacrificed at predetermined time points after administration and tissue samples are collected to pre-weighed tubes and measured on an automated gamma counter with weighed (approximately $10 \mu \mathrm{l}$ ) standards prepared from the formulated radiotracer solution. The output in biodistibution studies is the percentage of the injected (radioactive) dose per gram of tissue $(\% / D / g)$. The injected dose is determined using the standards when the weight of the administered formulation is known (from weighing the syringe before and after administration). Depending on the application, the ex vivo biodistribution studies can be preceded by or conducted alongside small animal PET or SPECT/CT imaging studies discussed next.

Autoradiography is a sensitive imaging technique to study the tissue-level distribution of radiolabeled tracers ex vivo in cryosections prepared from tissue samples. The autoradiographic image can then be overlaid to a histological or immunofluorescence staining image of the same or adjacent section to correlate the radioactivity accumulation for example with the presence of the target of the theranostic nanosystem or physiological change elicited by the nanosystem accumulation. Today, most autoradiographic systems employ digital imaging plates instead of conventional X-ray films to record the autoradiographic image. In the digital imaging plate, the energy emitted by the radioisotope is stored by a phosphor layer of europium-doped barium fluoroborate crystals and released through the excitation of the $\mathrm{Eu}^{2+}$ to unstable $\mathrm{Eu}^{3+}$ when the plate is scanned with a red (633 $\mathrm{nm}$ ) laser resulting in emission of blue light $(390 \mathrm{~nm})$ as the Eu returns to the ground state from the areas of the plate that were exposed to radiation. The emission is called photostimulated luminescence (PSL) and the quantification is often carried out in units of $\mathrm{PSL} / \mathrm{mm}^{2}$. Also real-time autoradiographic systems, where the tissue sample is encased in a parallel ionization multiplier (PIM) chamber are available. In these systems, the signal is accumulated as true counts or count rate per area, and the measurement time can be increased accordingly during the acquisition to improve the signal-to-background ratio in the image. In contrast, in digital autoradiography the autoradiographic trace is lost from the plate during the readout and in the case of short-lived isotopes only a single exposure will be possible.

\subsection{Small animal PET and SPECT/CT imaging studies}

Small animal PET and SPECT/CT imaging technology allows for the non-invasive imaging of radiotracer biodistribution in laboratory rodents. Imaging studies are typically conducted under inhalation anesthesia, and the commercial imaging systems contain solutions for the administration of anesthesia, warming the animals during the scan and nowadays also platforms for the imaging of multiple animals in a single scan. The power of imaging over the conventional ex vivo biodistribution studies described above is that the entire biodistribution - including organs that are not necessarily sampled for the gamma counting - can be seen in the image and thus possible unexpected accumulation will not go undetected. Furthermore, an individual animal can be followed up longitudinally using imaging, which reduces the number of animals needed for a study as well as allows for the account of inter-individual differences such as tumor heterogeneity or stage and disease progression on the study outcome. The ex vivo biodistribution studies are, however, usually needed at some point of radiotracer development to validate the results of the imaging and to provide quantification for images acquired with SPECT, a semi-quantitative method. Imaging can be carried out a dynamic acquisition protocol, where the imaging acquisition is started at the time of the radiotracer injection or immediately after to track the early passage of the radiotracer with sequentially acquired images typically over the first 60 minutes of 
injection. In static acquisition, the signal is collected over a fixed period of time or up to a desired number of counts giving a summed image of the radiotracer distribution. Most small animal imaging systems available today are hybrid systems, where an anatomical imaging modality such as CT or MRI is provided together with the nuclear imaging and workflows can be programmed for sequential acquisition of the two.

\subsection{Radiotherapy studies and dosimetry}

The radiotherapeutic effects of theranostic nanosystems are most commonly determined by following the tumor size and attainment of the experiment humane endpoints after the administration of the radiotherapy. This can be done by conventional caliper measurements for subcutaneous or superficial orthotopic tumors, or for example by optical imaging of orthotopic tumors if a luciferase or fluorescent protein -expressing cell line is used, by regular weighing of the animals, and by the use of a technique called body condition scoring[38] to monitor the condition of the animals. In addition, the effects of the radiation exposure on the blood values (such as red blood cell count, hematocrit, white blood cell and platelet counts) of the animals are commonly studied as an indicator for hematological toxicity and when comparing different dosing regimens[39]. Dedicated veterinary systems for blood analysis in the small volumes collected from laboratory rodents are available. Another important factor for the feasibility of the clinical translation of a theranostic nanosystem will be the dosimetry, i.e. the radioactive dose imparted by the nanosystem on the tumor and healthy tissues in the body. The dosimetry calculations are determined by measuring the time activity curves (TAC) for the clearance of the radiotracer in selected organs (the $\% \mathrm{ID} / \mathrm{g}$ in each can be determined either by imaging or ex vivo biodistribution) and fitting a typically one or two-phase decay equation in the data. The area under the curve then gives the cumulative uptake which can be converted to absorbed dose making the necessary assumptions for the absorbed fraction of the radiation and equilibrium absorbed dose constants depending on properties of the radioisotope. Human dosimetry for the same radiotracer can be extrapolated from the mouse data using a number of computational models and reference values such as those set forth by the International Commission for Radiation Protection (ICRP)[39-41].

\section{Current examples of radiolabeled theranostic nanosystems}

Theranostic nanoparticles combine both diagnostic and therapeutic agents in a single platform. Such dual imaging and therapy platforms allow imaging of the drug delivery, drug release and drug efficacy. The core of the theranostic nanosystems can be metallic, polymeric or lipid based such as micelles and liposomes. The surface of the nanosystem is modified to make it biocompatible and functionalized such that it can attach biological targeting agents, imaging or therapeutic molecules. The surface modifications allow prolongation of plasma half-life, alteration of pharmacokinetics (PK) and biodistribution (BD), suppression of immunogenicity and antigenicity, stabilization against enzymatic degradation, reduced efflux of drugs, improved cellular endocytosis, and change in solubility characteristics. Multimodal imaging is often used since the precision and sensitivity of detection varies across modalities based on the size and location of the tumor or metastasis.[42] Among the imaging modalities of MR, optical, SPECT, PET and CT the combinations are chosen such that ideally anatomical, physiological and molecular information is acquired with high sensitivity as illustrated by Figure 23. 


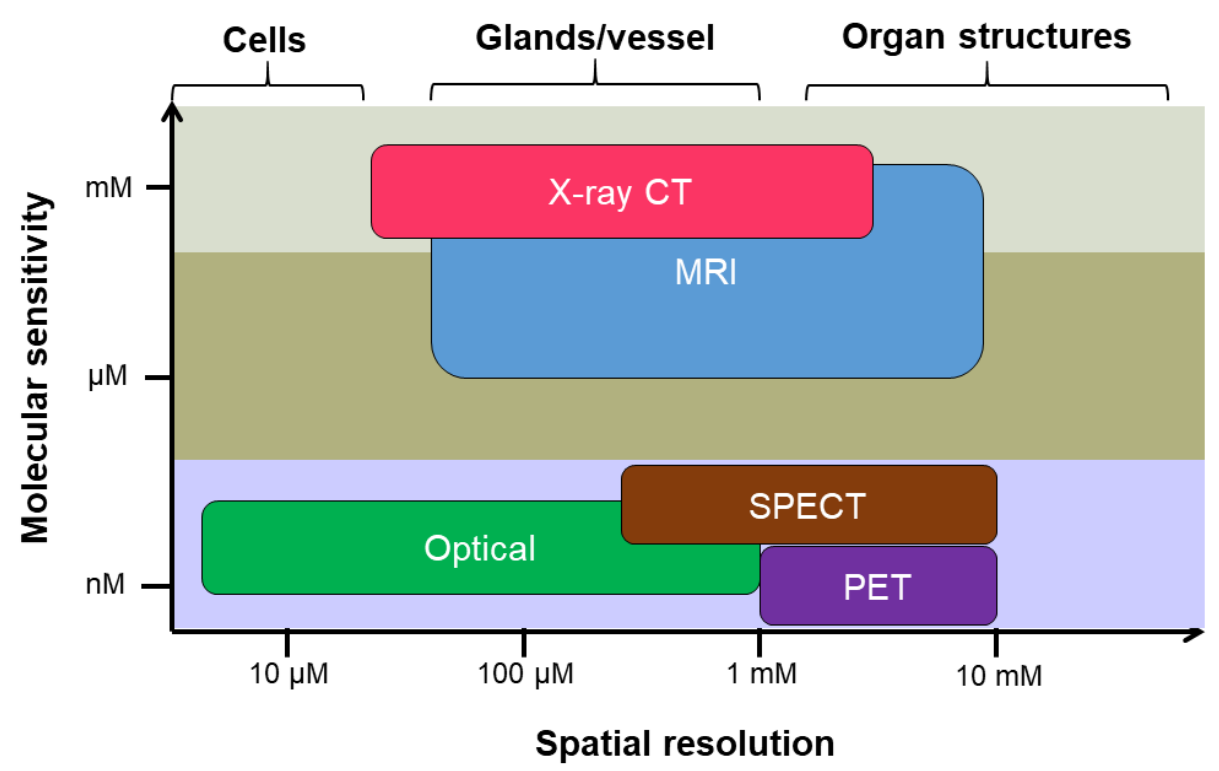

Figure 23. Precision and sensitivity of detection varies across imaging modalities.

While passive targeting is more suited for fast growing tumors and nanoparticles which have a long circulation time, most tumors have unpredictable extravasation of the nanoparticle with varied sizes, shapes and surface charge. Hence, targeting groups allow specific targeting beyond the effects of enhanced permeating and retention (EPR). This is particularly important in poorly vascularized small metastasis which are below $100 \mathrm{~mm}^{3}$.[4345]

Therapeutic agents loaded onto the nanoparticle can be released passively or with the stimuli of light or tumor microenvironment. In certain types of therapy, the external stimuli directly modulate the therapy such in laser assisted ablation therapy. Well known examples of such therapies are photodynamic therapy (PDT) and photothermal therapy (PTT) where the light stimuli activates the photosensitizer which could be a small molecule or a nanoparticle. The imaging techniques used are positron emission tomography (PET), singlephoton emission computed tomography (SPECT), computed tomography (CT), magnetic resonance imaging $(\mathrm{MRI})$, optical/photoacoustic imaging and contrast enhanced ultrasound.[46, 47]

Diagnostic and therapeutic radioisotopes such as copper-64 and lutetium-177 can be bound to the same DOTA chelator. They have been loaded onto DOTA containing liposomes and used for PET imaging and therapy. Using PET imaging the optimum PEGylation and its influence on the biodistribution and tumor accumulation can be determined. A high therapeutic dose $(114 \mathrm{mGy} / \mathrm{MBq})$ could be delivered to the tumor site for the lutetium-177 loaded liposomes.[48] Liposomal formulations using doxorubicin as a chemothereutic agent in combination with rhenium-188 which has therapeutic properties as well SPECT imaging capabilities have been shown to greatly inhibit tumor growth and enhance median survival time in a murine colon carcinoma model. The therapeutic results were better than singly functionalized rhenium-188-liposomes or doxorubicin-liposomes which shows that the synergistic effects of chemotherapy and internal radiation therapy are far more effective in therapy.[49] Multifunctional liposomes have been reported which have been loaded with doxorubicin for therapy, gadolinium ions for MR imaging, IRDye for NIR 
fluorescence imaging, technetium-99m for SPECT imaging and copper-64 for PET imaging. In these DSPC-based liposomes, the drug was incapsulated, technetium-99m was post loaded while the copper-64 was conjugated to the DOTA chelators available on the liposome surface. The liposomes showed effective and correlated multimodal imaging capabilities in head and neck cancer mice model.[50] The drug delivery and therapeutic response was quantified in a clinical study with copper-64 labeled HER2 targeted PEGylated liposomal doxorubicin (Doxil). This study provided evidence that the EPR effect is functional in human tumor and imaging liposomal deposition can identify patients who are ideal for receiving therapeutic liposomes. The tumor concentration, rates of deposition and washout of the liposomes in human tumor compared well to preclinical models, which is of high significance to future studies.[51] Such image-guided insights are useful to prescreen patients assigned to Doxil in order to identify which patients are likely respond to therapy and which are not. The release of a doxorubicin from liposomes could be visualized using MRI by using MR contrast agents such as manganese ions which were mixed with doxorubicin and upon simultaneous liberation enhanced contrast due to increase in water exchange rates. Other studies with MRI contrast agents to follow drug release have used ProHance (Gd-HPDO3A).[52, 53] Alpha particles have short penetration depth and high linear energy transfer (LET) which gives it an advantage over beta or gamma radiation. Alpha particle emitters such as ${ }^{225} \mathrm{Ac},{ }^{223} \mathrm{Ra}$ and ${ }^{224} \mathrm{Ra}$ have made it to the clinic. The limitations associated with alpha emitters are that the daughter ions when not retained at the tumor site can cause significant damage to healthy tissue. ${ }^{211} \mathrm{Bi}$ causes renal toxicity which is a limiting factor for performing clinical studies. The ${ }^{225} \mathrm{Ac}$ could be doped into the core of a nanoparticle prepared from lanthanum chloride, gadolinium chloride and cross-linked with sodium tripolyphosphate. The core nanoparticle is then covered with layers of gadolinium phosphate and gold. The magnetic properties of gadolinium phosphate allow easy separation and the gold surface provides biocompatibility and easy attachment of targeting moieties. The multilayered structure allowed $99.9 \%$ retention of ${ }^{225} \mathrm{Ac}$ and $98 \%$ retention of daughter ions. The gamma rays from the ${ }^{221} \mathrm{Fr}$ allow SPECT/CT imaging. The nanoparticles with antibodies targeted to the thrombomodulin receptors of lung showed predominant lung uptake $1 \mathrm{~h}$ post injection.[54]

Carbon based nanomaterials include fullerenes, carbon nanotubes, graphenes and carbon-based quantum dots. The intrinsic property of one-photon and two-photon fluorescence in the NIR II allows deep tissue optical imaging. Their biocompatibility and ease of functionalization make them interesting candidates for theranostics applications. $A{ }^{64} \mathrm{Cu}$ labeled doxorubicin loaded polydopamine-gadolinium-metallofullerene $\left(\mathrm{Gd}_{3} \mathrm{~N} @ \mathrm{C}_{80}\right)$ coresatellite nanotheranostics was prepared which was capable of MR/photoacoustic/PET imaging and NIR triggered drug delivery in combination with PTT. The gadolinium showed high T1 contrast and was retained well in the particles. The doxorubicin was bound to the polydopamine through $\pi-\pi$ stacking and hydrogen-bonding interactions, which were disrupted with NIR, causing the release of doxorubicin. The PTT caused the tumor temperature to reach to $46{ }^{\circ} \mathrm{C}$ with $808 \mathrm{~nm}$ laser in $10 \mathrm{~min}$ and the combination therapy slowed the tumor growth and was significantly more effective than the monotherapy.[55] Single wall carbon nanotube (SWCNT) have inherent Raman signature which allows the direct monitoring of the presence of nanotubes in mice tissue. They were covalently attached to DOTA or DFO to chelate to actinium-225 or zirconium-89, respectively. The SWCNT was attached to tumor neovascular-targeting antibody E4G10. The alpha particles from ${ }^{225} \mathrm{Ac}$ have a travelling distance which match the vessel dimensions and delivers high linear energy transfer (LET) to the cells resulting in acute cytotoxicity making it ideal vascular- 
targeted radioimmunotherapy. The ${ }^{89} \mathrm{Zr}$ labeled constructs allowed PET imaging and determination of pharmacokinetics of the construct.[56] PET imaging and dosimetry of ${ }^{90} \mathrm{Y}$ SIR-Sphere using ${ }^{86} \mathrm{Y}$ and ${ }^{89} \mathrm{Zr}$ radiolabeled surrogates could be performed, and showed in vivo stability for clinical application. Theranostic application could be easily envisioned with the similarity in half lives of ${ }^{89} \mathrm{Zr}$ and ${ }^{90} \mathrm{Y}[57]$.

Gold nanoparticles can be prepared in different geometries, such as nanospheres, nanoshells, nanorods, or nanocages, which along with size effect the photothermal conversion efficiency for photothermal therapy. The absorbed wavelength changes depending on the shape of the particle so it has to be tuned such that it absorbs NIR in the two biologically transparent optical windows available, $650-850 \mathrm{~nm}$ and $950-1350 \mathrm{~nm}$. The gold surface is ideal for binding to free thiol which makes it an ideal choice for bioconjugation. Copper sulphide nanoparticles are advantageous to gold nanoparticles as they are considered biodegradable inorganic nanomaterials, the surrounding environment does not affect its absorption wavelength and lastly the cost of production is much lower. CuS nanoparticles were coupled to ${ }^{64} \mathrm{Cu}$ using chelator-free methods and used for PTT therapy with 800 and $980 \mathrm{~nm}$ laser showing better photothermal effects with $980 \mathrm{~nm}$ laser. The photothermal conversion efficiencies of CuS nanoparticles were reported to be much higher than gold nanospheres and SWCNTs with $980 \mathrm{~nm}$ laser reaching $99.85{ }^{\circ} \mathrm{C}$ at 2 $\mathrm{W} / \mathrm{cm}^{2}$ for $4 \mathrm{~min}$. The tumor distribution of the particles was assessed using PET imaging and image guided PTT could be performed with thermal mapping system equipped MRI scanner. In the orthotopic ovarian cancer mice model, this resulted in effective therapeutic outcome.[58] To improve on the previous results in terms of the tumor availability of the nanoparticle, RGDfK peptides were attached to the CuS nanoparticles to target the av $\beta 3$ integrins and labelled with ${ }^{64} \mathrm{Cu}$ in a chelator-free fashion as before. For the therapy a lower wavelength of $808 \mathrm{~nm}$ at $3 \mathrm{~W} / \mathrm{cm}^{2}$ for 2 min was used which increased the tumor temperature to $58.1{ }^{\circ} \mathrm{C}$ and resulted in $98 \%$ tumor tissue necrosis in U87 tumor-bearing mice.[59] Similar constructs can be used to target other biomarkers such as folate receptors, which are overexpressed in ovarian, breast, lunch and head and neck cancers.[60] CuS nanoparticle microspheres were loaded with chemotherapeutic paclitexel and radiolabeled with ${ }^{131}$ I. These were used for PTT, chemotherapy, radiotherapy, photoacoustic imaging and SPECT/CT from a single construct. The trimodal combination therapy with NIR irradiation eliminated tumor growth after intratumoral injection at relatively low doses in orthotopic breast cancer model as shown in Figure 24[61]. Theranostic Gd:CuS@BSA nanoparticle were prepared using copper(II) sulphide and gadolinium(III) ions with bovine serum albumin (BSA) as scaffold. The $9 \mathrm{~nm}$ particles which showed high photothermal conversion efficiency and good photostability under near-infrared (NIR) laser irradiation. Mice with ovarian cancer xenograft showed tumor growth inhibition with 5 min irradiation using $980 \mathrm{~nm}$ laser irradiation with tumor temperature going up to $50{ }^{\circ} \mathrm{C}$.[62] Defect-rich multifunctional Cudoped layered double hydroxide (Cu-LDH) nanoparticles were shown to have $\mathrm{pH}$ enhanced NIR PTT as well as $\mathrm{pH}$-sensitive T1 weighted MRI properties. The peculiar microstructures gave these nanoparticles high photothermal conversion at $808 \mathrm{~nm}$ and $\mathrm{pH}$ sensitivity. Further these particles were loaded with chemotherapeutic agent 5-FU to combine PTT with chemotherapy and dose dependent cytotoxicity was observed. Mice with colon cancer xenografts could be MR imaged and showed complete ablation with the combination of PTT and chemotherapy.[63] 

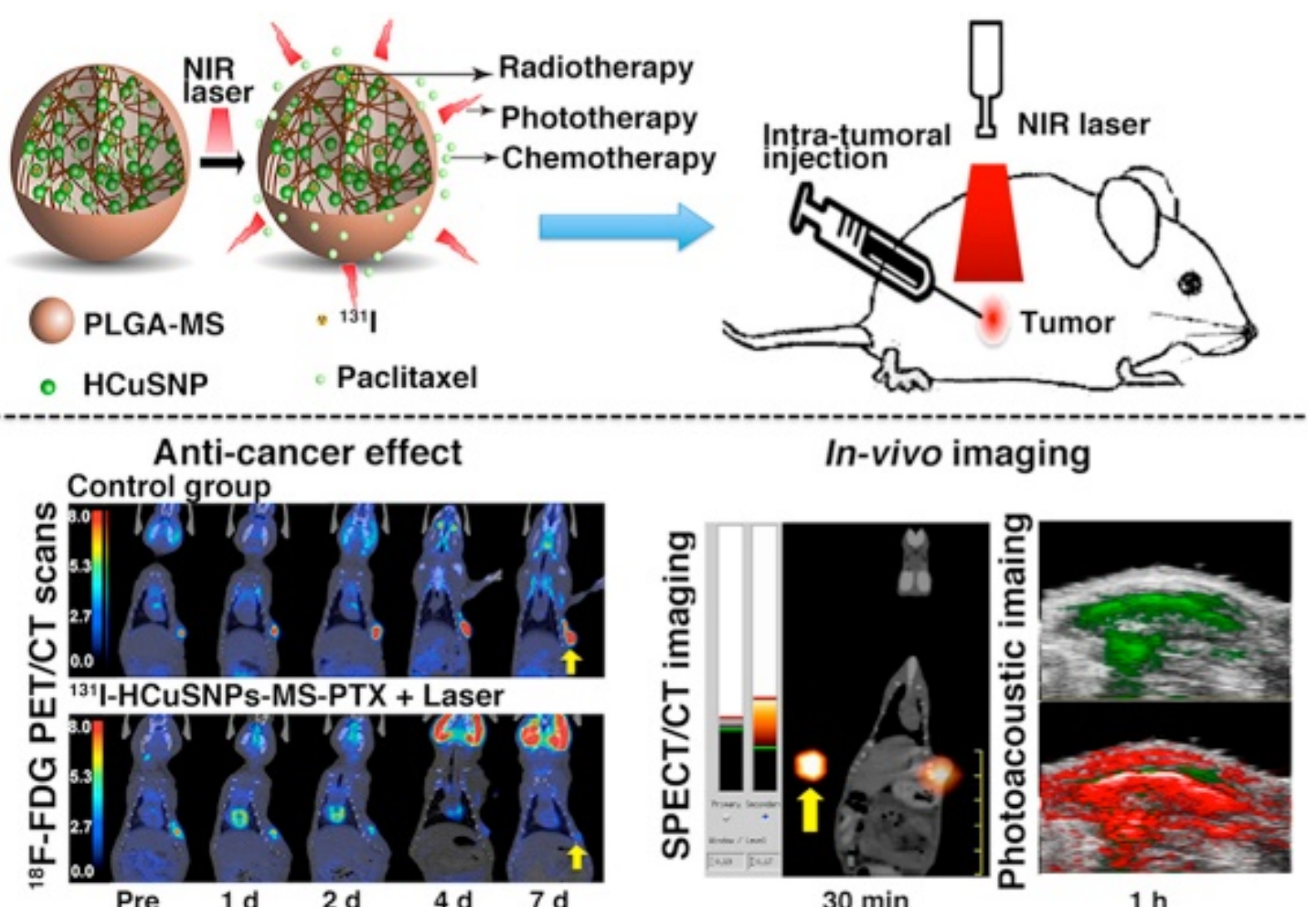

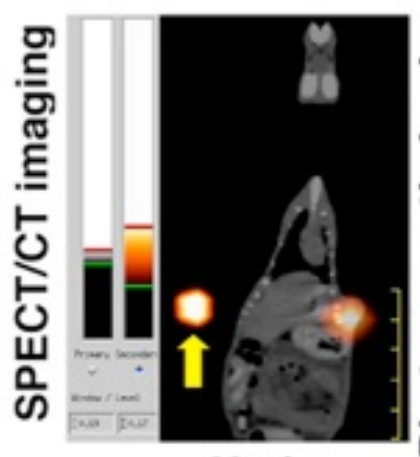

$30 \mathrm{~min}$

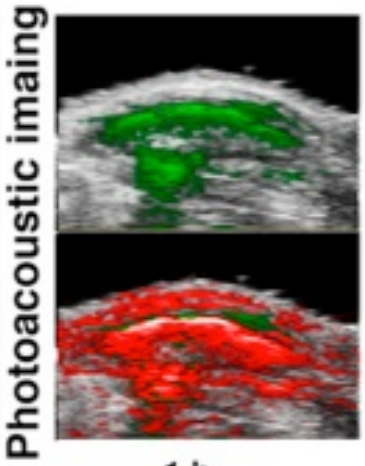

$1 \mathrm{~h}$

Figure 24. Multimodal imaging and combination therapy using ${ }^{131}$ l-labeled CuS based particles. Figure reprinted with permission from reference [62].

A melanin-based nanosystem with inherently good biocompatibility, biodegrability and intrinsic photoacoustic properties was functionalized with tyrosine kinase inhibitor, sorafenib using $\pi-\pi$ interaction. In addition, these particles had the natural ability to bind ${ }^{64} \mathrm{Cu}$ or ferric ions for PET or MR imaging studies, respectively as shown in Figure 25. PET imaging allowed accurate and sensitive measurement of tissue penetration of the agent whereas the photoacoustic imaging allowed superficial information due to limitations of the penetrability of NIR laser. Significant tumor shrinkage was observed from a single dose of the agent.[64, 65]
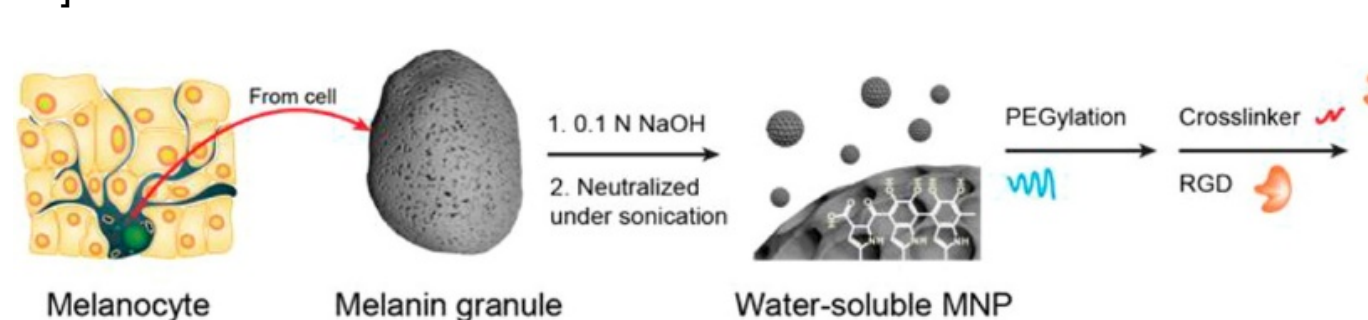

Melanocyte

Melanin granule

Water-soluble MNP

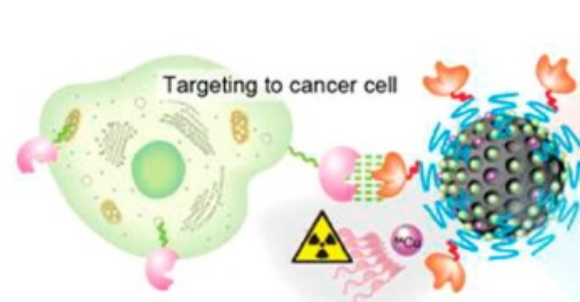

PET

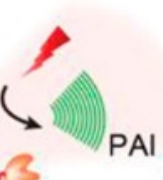

(1)

MRI 
Figure 25. An overview of the synthesis and surface modification of melanin nanoparticles and subsequent chelation to ${ }^{64} \mathrm{Cu}$. Figure reprinted with permission from reference [65].

Water-soluble superparamagnetic iron oxide (SPIO) nanoparticles such as ferumoxides and ferucarbotrans are clinically approved for MR imaging of the liver diseases. The approval of the SPIO's make them potential candidates for further approval as drug delivery candidates. PEGylated SPIO's were functionalized with cRGD for targeted tumor delivery. They were conjugated with ${ }^{64} \mathrm{Cu}$ using NOTA as chelator for PET imaging. The $\mathrm{pH}$ sensitive drug delivery was achieved using hydrazone linked doxorubicin which showed effective release at $\mathrm{pH}$ 5.3. Quantifying the PET images showed that targeting the SPIO's with cRGD resulted in doubling of nanoparticle delivery.[66]

The drug release from the nanoparticle can be triggered by various external stimuli such as ultrasound, light, thermal, or chemical environment changes. The success of the PDT based treatment is not without its drawbacks. Since the photosensitizers are always in an "ON" state, patients are typically vulnerable to sunlight even after 4-6 weeks of the PDT therapy. Also the hypoxic conditions of the tumor microenvironment hinders PDT therapy due to its inherent requirement for oxygen to generate reactive oxygen species. $A$ nanoparticle (PcS-MA) formed from the host-guest interactions of water-soluble photosensitizer, zinc(II) phthalocyanine tetrasubstituted with 6,8-disulfonate-2-naphthyloxy groups, and the common anticancer drug, mitoxantrone (MA) were quenched in supramolecular structure and showed no PDT effect or fluorescence signal. Upon interaction with nucleic acids, the construct disassembles resulting in PDT and chemotherapy, and could be imaged using fluorescence. MCF7 tumor-bearing mice which were injected with PcS-MA and irradiated with $1 \mathrm{~W} / \mathrm{cm}^{2}$ for 5 min reached at maximum temperature of $42.7^{\circ} \mathrm{C}$ at the tumor site and was able inhibit tumor growth. Thus, such an activatable theranostic strategy could circumvent the drawbacks of traditional PDT in clinical settings.[63]

The onset of hypoxia from PDT has been used as a trigger for a hypoxia activated prodrug AQ4N. The liposomal system was prepared by encapsulating AQ4N and hexadecylamine conjugated $\mathrm{Ce} 6$ (hCe6) photosensitizer in PEGylated liposomes which had a size of $95 \mathrm{~nm}$. Conjugating the hCe6 with a positron emitter like ${ }^{64} \mathrm{Cu}$ allowed PET imaging along with the inherently available fluorescence and optoacoustic imaging. All three modalities allowed the visualization of the liposomal delivery in the breast cancer model in mice with PET being the most sensitive of them all as expected. Biodistribution studies with the PET tracer also allowed quantification of the delivered dose. The therapy regiment showed that the synergistic effects of PDT and the hypoxia driven drug activation resulted in stagnation of the tumor growth. [67]

Delivery of the drug to the tumor site is a challenge due to the insufficient accumulation of the nanoparticle. The size of the nanoparticle has to be optimized to have desired blood circulation time. Other constraints which favor small particle size are inefficient tumor vascular extravasation, high interstitial fluid pressure and dense extracellular matrix. Nanoparticles with smaller size are hence desired because of their high diffusion to the tumor tissue but a size less than $5 \mathrm{~nm}$ results in rapid excretion through the kidneys. Another crucial factor is the charge of the particles which is preferred to be positive for internalization whereas immune processes like opsonization can easily recognize these positively charged particles and eliminate from the blood stream. Xue et al. developed upPhD theranostic nanovehicles, which had a dual size and charge transformability from the acidic $\mathrm{pH}$ of the tumor microenvironment. The monomers $(\mathrm{PhD})$ were synthesized by conjugating cytotoxic doxorubicin and PDT agent, pheophorbide using $\mathrm{pH}$ sensitive hydrazone bonds. The 
monomers first self-assembled into ultra-small micelles (upPhD) of about $4 \mathrm{~nm}$ in size. The upPhD's were then pegylated using $\mathrm{pH}$ sensitive imine bonds to form particles (pPhD) with $79 \mathrm{~nm}$ in size. When pPhD's were exposed to acidic $\mathrm{pH}$, hydrolysis of the pegylation resulted in reverting to the smaller sized upPhD particles. The strong positive charge of upPhD (43 $\mathrm{mV}$ ) was reduced to $12 \mathrm{mV}$ upon formation of the pegylated $\mathrm{pPhD}$ particles at neutral $\mathrm{pH}$ but reverted back to $35 \mathrm{mV}$ at $\mathrm{pH}$ 6.8. The fluorescence of both the monomer components were also quenched in $\mathrm{pPhD}$ particles but was regained at a $\mathrm{pH}$ of 6.8. In subcutaneous and orthotropic tumor mice models, the pPhD particles showed $100 \%$ cure rate and outperformed the upPhD particles showing the importance of charge and size modulation. The large, slightly positive nanovehicles had long circulation time but could be broken down into ultra-small nanoparticles with high positive charge near the tumor microenvironment, ideal for cell penetration. The particle accumulation at the tumor site could be followed using the fluorescence signal of the monomers as well as by MR imaging of manganese ions bound to the PDT agent. [68]

Biomimetic design strategies such as the ones using cell derived membranes synthesize natural vesicles are particularly interesting due to their excellent biocompatibility, low toxicity, high tumor accumulation and ability to evade the immune and reticuloendothelial systems. Multicompartment membrane-derived liposomes (MCLs) were prepared using 4T1 breast cancer cell membranes fused with surfactant, Tween-80, and had a size of $140 \mathrm{~nm}$ which was considerably smaller than without Tween-80. As illustrated in Figure 26, quantitative biodistribution using DFO conjugated ${ }^{89} \mathrm{Zr}$ showed greater than $9 \% \mathrm{ID} / \mathrm{g}$ in $4 \mathrm{~T} 1$ breast cancer tumor model in mice and clearance mostly though liver. Injection to the lymphatic system allowed imaging of the lymph nodes. PDT agent loaded liposomes showed reduction in the rate of growth of the tumor upon irradiation with $660 \mathrm{~nm}$ laser.[69] 
A
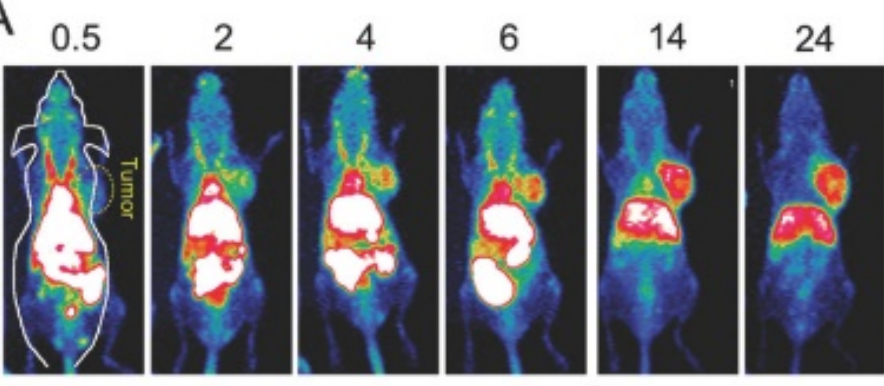

48

$72 \mathrm{~h}$

B
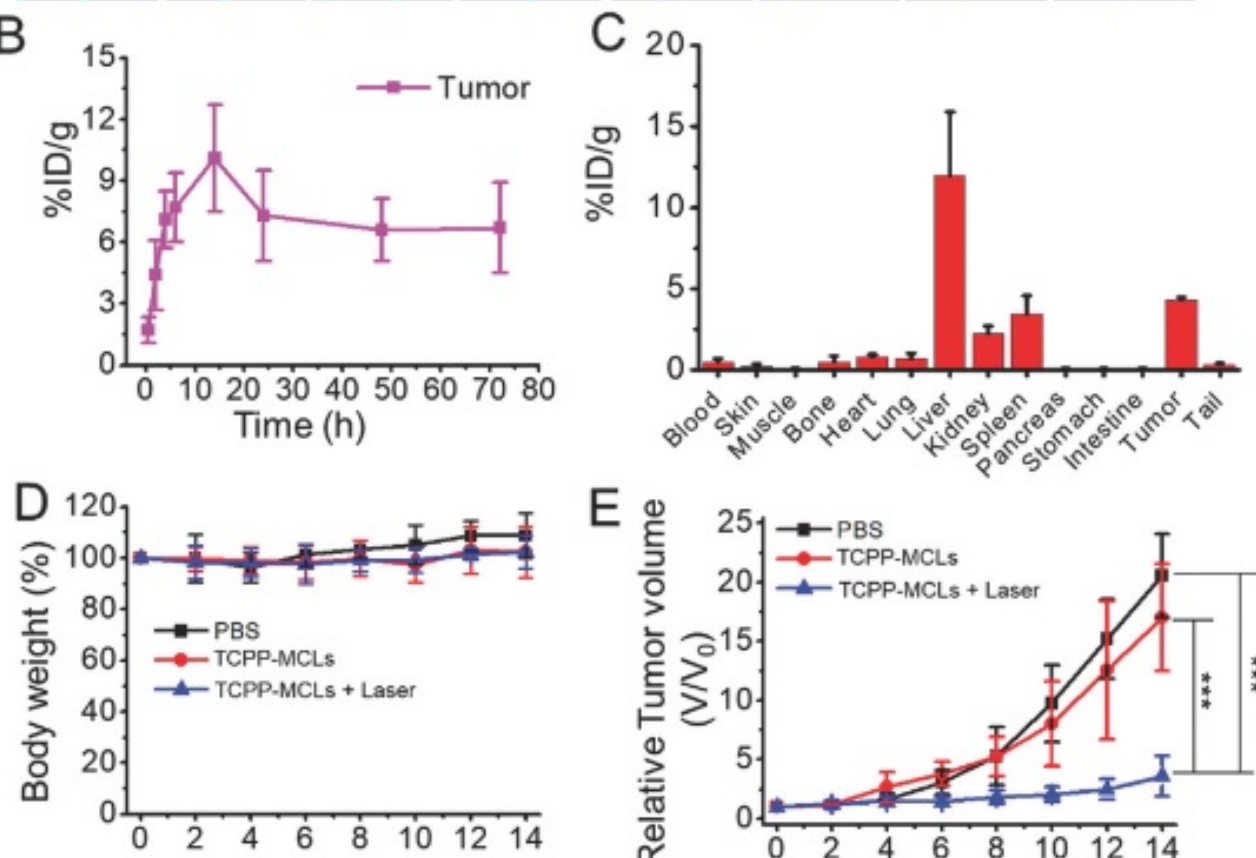

E

Days after treatment

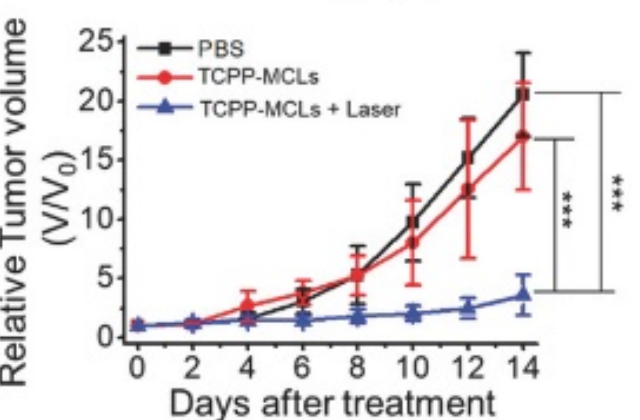

Figure 26. Imaging and therapy using multicompartment membrane-derived liposomes (MCLs) A) In vivo PET images of $4 \mathrm{~T} 1$ bearing mice at various time intervals after injection of ${ }^{89} \mathrm{Zr}$ labeled MCLs B) Tumor uptake values at different time points C) $72 \mathrm{~h}$ biodistribution profile in various organs D) Mice body weight during various treatments E) 4T1 tumor growth profiles with mice treated with $660 \mathrm{~nm}$ laser $(50 \mathrm{~mW} \mathrm{~cm}-2,40 \mathrm{~min})$ at $24 \mathrm{~h}$ post injection with corresponding controls. Figure reprinted with permission from reference [69].

\section{References}

[1] Aghevlian S, Boyle AJ, and Reilly RM. Radioimmunotherapy of cancer with high linear energy transfer (LET) radiation delivered by radionuclides emitting $\alpha$-particles or Auger electrons. Adv Drug Delivery Rev 2017;109:102-18.

[2] Eckelman WC, Boyd M, and Mairs RJ. Principles of Molecular Targeting for Radionuclide Therapy. In: HW Strauss, G Mariani, D Volterrani, and SM Larson editors. Nuclear Oncology: From Pathophysiology to Clinical Applications. Cham: Springer International Publishing; 2017, p. 35-65.

[3] Schirrmacher R, Wängler B, Bailey J, Bernard-Gauthier V, Schirrmacher E, and Wängler C. Small Prosthetic Groups in 18 F-Radiochemistry: Useful Auxiliaries for the Design of 18 F-PET Tracers. Seminars in Nuclear Medicine 2017;47:474-92.

[4] Coenen HH, Gee AD, Adam M, Antoni G, Cutler CS, Fujibayashi Y, et al. Consensus nomenclature rules for radiopharmaceutical chemistry - Setting the record straight. Nucl Med Bio 2017;55:v-xi.

[5] Bergström M, Grahnén A, and Långström B. Positron emission tomography microdosing: a new concept with application in tracer and early clinical drug development. European journal of clinical pharmacology 2003;59:357-66. 
[6] Price EW and Orvig C. Matching chelators to radiometals for radiopharmaceuticals. Chem Soc Rev 2014;43:260-90.

[7] Zhou M, Chen Y, Adachi M, Wen X, Erwin B, Mawlawi O, et al. Single agent nanoparticle for radiotherapy and radio-photothermal therapy in anaplastic thyroid cancer. Biomaterials 2015;57:41-9.

[8] Uccelli L, Martini P, Pasquali M, and Boschi A. Monoclonal Antibodies Radiolabeling with Rhenium-188 for Radioimmunotherapy. Biomed Res Int 2017;2017:5923609.

[9] Yuan H, Wilks MQ, El Fakhri G, Normandin MD, Kaittanis C, and Josephson L. Heat-induced-radiolabeling and click chemistry: A powerful combination for generating multifunctional nanomaterials. PLoS One 2017;12:e0172722.

[10] Boros E, Bowen AM, Josephson L, Vasdev N, and Holland JP. Chelate-free metal ion binding and heatinduced radiolabeling of iron oxide nanoparticles. Chem Sci 2015;6:225-36.

[11] Hoffman D, Sun M, Yang L, McDonagh PR, Corwin F, Sundaresan G, et al. Intrinsically radiolabelled [(59)Fe]-SPIONs for dual MRI/radionuclide detection. Am J Nucl Med Mol Imaging 2014;4:548-60.

[12] Shi S, Fliss BC, Gu Z, Zhu Y, Hong H, Valdovinos HF, et al. Chelator-Free Labeling of Layered Double Hydroxide Nanoparticles for in Vivo PET Imaging. Sci Rep 2015;5:16930.

[13] Shi S, Xu C, Yang K, Goel S, Valdovinos HF, Luo H, et al. Chelator-Free Radiolabeling of Nanographene: Breaking the Stereotype of Chelation. Angew Chem Int Ed Engl 2017;56:2889-92.

[14] Jakobsson U, Mäkilä E, Airaksinen AJ, Alanen O, Etilé A, Köster U, et al. Porous Silicon as a Platform for Radiation Theranostics Together with a Novel RIB-Based Radiolanthanoid. Contrast Media \& Molecular Imaging 2019:3728563.

[15] Kabalka GW and Yao M-L. No-carrier-added radiohalogenations utilizing organoboranes: The synthesis of iodine-123 labeled curcumin. Journal of Organometallic Chemistry 2009;694:1638-41.

[16] Ishiyama T, Murata M, and Miyaura N. Palladium(0)-Catalyzed Cross-Coupling Reaction of Alkoxydiboron with Haloarenes: A Direct Procedure for Arylboronic Esters. The Journal of Organic Chemistry 1995;60:7508-10.

[17] Hunter R. Standardization of the Chloramine-T Method of Protein lodination. Experimental Biology and Medicine 1970;133:989-92.

[18] Conlon JM. The use of IODO-GEN for preparing 125I-labeled peptides and their purification by reversed-phase high performance liquid chromatography. Methods Mol Biol 1997;73:231-7.

[19] Krohn KA, Knight LC, Harwig JF, and Welch MJ. Differences in the sites of iodination of proteins following four methods of radioiodination. Biochimica et Biophysica Acta (BBA) - Protein Structure 1977;490:497-505.

[20] Bolton AE and Hunter WM. The labelling of proteins to high specific radioactivities by conjugation to a125I-containing acylating agent. Application to the radioimmunoassay. Biochemical Journal 1973;133:52938.

[21] Sloan NL, Luthra SK, McRobbie G, Pimlott SL, and Sutherland A. A one-pot radioiodination of aryl amines via stable diazonium salts: preparation of 1251-imaging agents. Chemical Communications 2017;53:11008-11.

[22] Cauchon N, Langlois R, Rousseau JA, Tessier G, Cadorette J, Lecomte R, et al. PET imaging of apoptosis with $64 \mathrm{Cu}$-labeled streptavidin following pretargeting of phosphatidylserine with biotinylated annexin-V. European Journal of Nuclear Medicine and Molecular Imaging 2007;34:247-58.

[23] Honarvar H, Westerlund K, Altai M, Sandström M, Orlova A, Tolmachev V, et al. Feasibility of Affibody Molecule-Based PNA-Mediated Radionuclide Pretargeting of Malignant Tumors. Theranostics 2016;6:93103.

[24] van Rij CM, Frielink C, Goldenberg DM, Sharkey RM, Franssen GM, Lütje S, et al. Pretargeted ImmunoPET of Prostate Cancer with an Anti-TROP-2 x Anti-HSG Bispecific Antibody in Mice with PC3 Xenografts. Mol Imaging Biol 2014;17:94-101.

[25] Keinänen O, Mäkilä EM, Lindgren R, Virtanen H, Liljenbäck H, Oikonen V, et al. Pretargeted PET Imaging of trans-Cyclooctene-Modified Porous Silicon Nanoparticles. ACS Omega 2017;2:62-9. 
[26] Cędrowska E, Pruszynski M, Majkowska-Pilip A, Męczyńska-Wielgosz S, Bruchertseifer F, Morgenstern A, et al. Functionalized TiO2 nanoparticles labelled with 225Ac for targeted alpha radionuclide therapy. Journal of Nanoparticle Research 2018;20.

[27] Piotrowska A, Męczyńska-Wielgosz S, Majkowska-Pilip A, Koźmiński P, Wójciuk G, Cędrowska E, et al. Nanozeolite bioconjugates labeled with 223 Ra for targeted alpha therapy. Nuclear Medicine and Biology 2017;47:10-8.

[28] Wang G, de Kruijff RM, Rol A, Thijssen L, Mendes E, Morgenstern A, et al. Retention studies of recoiling daughter nuclides of 225Ac in polymer vesicles. Applied Radiation and Isotopes 2014;85:45-53.

[29] Guerard F, Navarro L, Lee YS, Roumesy A, Alliot C, Cherel M, et al. Bifunctional aryliodonium salts for highly efficient radioiodination and astatination of antibodies. Bioorg Med Chem 2017;25:5975-80.

[30] Teiluf K, Seidl C, Blechert B, Gaertner FC, Gilbertz K-P, Fernandez V, et al. $\alpha$-Radioimmunotherapy with $213 \mathrm{Bi}$-anti-CD38 immunoconjugates is effective in a mouse model of human multiple myeloma. 2014;6.

[31] Kuo L and Yang L-X. Y-H2AX - A Novel Biomarker for DNA Double-strand Breaks. In Vivo 2008;22:305-9. [32] Graf F, Fahrer J, Maus S, Morgenstern A, Bruchertseifer F, Venkatachalam S, et al. DNA double strand breaks as predictor of efficacy of the alpha-particle emitter Ac-225 and the electron emitter Lu-177 for somatostatin receptor targeted radiotherapy. PLoS One 2014;9:e88239.

[33] Haimovitz-Friedman A, Yang TJ, Thin TH, and Verheij M. Imaging radiotherapy-induced apoptosis. Radiat Res 2012;177:467-82.

[34] Wang Y, Boerma M, and Zhou D. Ionizing Radiation-Induced Endothelial Cell Senescence and Cardiovascular Diseases. Radiat Res 2016;186:153-61.

[35] Lee S and Schmitt CA. The dynamic nature of senescence in cancer. Nat Cell Biol 2019;21:94-101.

[36] Campisi J and d'Adda di Fagagna F. Cellular senescence: when bad things happen to good cells. Nature Reviews Molecular Cell Biology 2007;8:729.

[37] Iliakis G, Wang Y, Guan J, and Wang H. DNA damage checkpoint control in cells exposed to ionizing radiation. Oncogene 2003;22:5834-47.

[38] Ullman-Cullere $\mathrm{MH}$ and Foltz CJ, Body condition scoring: a rapid and accurate method for assessing health status in mice. 1999, p. 319-24.

[39] Poty S, Carter LM, Mandleywala K, Membreno R, Abdel-Atti D, Ragupathi A, et al. Leveraging Bioorthogonal Click Chemistry to Improve ${ }^{225}$ Ac-Radioimmunotherapy of Pancreatic Ductal Adenocarcinoma. Clin Cancer Res 2019;25:868-80.

[40] Keinänen O, Brennan JM, Membreno R, Fung K, Gangangari K, Dayts EJ, et al. Dual Radionuclide Theranostic Pretargeting. Mol Pharmaceutics 2019;16:4416-21.

[41] Stabin MG and Siegel JA. Physical models and dose factors for use in internal dose assessment. Health Physics 2003;85:294-310.

[42] Gao X, Guo L, Li J, Thu HE, and Hussain Z. Nanomedicines guided nanoimaging probes and nanotherapeutics for early detection of lung cancer and abolishing pulmonary metastasis: Critical appraisal of newer developments and challenges to clinical transition. Journal of Controlled Release 2018;292:29-57. [43] Maeda H, Sawa T, and Konno T. Mechanism of tumor-targeted delivery of macromolecular drugs, including the EPR effect in solid tumor and clinical overview of the prototype polymeric drug SMANCS. Journal of Controlled Release 2001;74:47-61.

[44] Allen TM. Drug Delivery Systems: Entering the Mainstream. Science 2004;303:1818-22.

[45] Chen F, Ehlerding EB, and Cai W. Theranostic Nanoparticles. Journal of Nuclear Medicine 2014;55:1919-22.

[46] Jokerst JV and Gambhir SS. Molecular imaging with theranostic nanoparticles. Acc Chem Res 2011;44:1050-60.

[47] Zavaleta C, Ho D, and Chung EJ. Theranostic Nanoparticles for Tracking and Monitoring Disease State. SLAS Technol 2018;23:281-93.

[48] Petersen AL, Henriksen JR, Binderup T, Elema DR, Rasmussen PH, Hag AM, et al. In vivo evaluation of PEGylated 64Cu-liposomes with theranostic and radiotherapeutic potential using micro PET/CT. European Journal of Nuclear Medicine and Molecular Imaging 2015;43:941-52. 
[49] Chang Y-J, Chang C-H, Yu C-Y, Chang T-J, Chen L-C, Chen M-H, et al. Therapeutic efficacy and microSPECT/CT imaging of 188Re-DXR-liposome in a C26 murine colon carcinoma solid tumor model. Nuclear Medicine and Biology 2010;37:95-104.

[50] Li S, Goins B, Zhang L, and Bao A. Novel Multifunctional Theranostic Liposome Drug Delivery System: Construction, Characterization, and Multimodality MR, Near-Infrared Fluorescent, and Nuclear Imaging. Bioconjugate Chem 2012;23:1322-32.

[51] Lee H, Shields AF, Siegel BA, Miller KD, Krop I, Ma CX, et al. (64)Cu-MM-302 Positron Emission Tomography Quantifies Variability of Enhanced Permeability and Retention of Nanoparticles in Relation to Treatment Response in Patients with Metastatic Breast Cancer. Clin Cancer Res 2017; 23:4190-202.

[52] Viglianti BL, Abraham SA, Michelich CR, Yarmolenko PS, MacFall JR, Bally MB, et al. In vivo monitoring of tissue pharmacokinetics of liposome/drug using MRI: Illustration of targeted delivery. Magnetic Resonance in Medicine 2004;51:1153-62.

[53] Lammers T, Aime S, Hennink WE, Storm G, and Kiessling F. Theranostic Nanomedicine. Accounts of Chemical Research 2011;44:1029-38.

[54] Han Z, McLaughlin MF, Woodward J, Boll RA, Wall JS, Rondinone AJ, et al. Gold Coated Lanthanide Phosphate Nanoparticles for Targeted Alpha Generator Radiotherapy. PLoS ONE 2013;8.

[55] Wang S, Lin J, Wang Z, Zhou Z, Bai R, Lu N, et al. Core-Satellite Polydopamine-GadoliniumMetallofullerene Nanotheranostics for Multimodal Imaging Guided Combination Cancer Therapy. Advanced Materials 2017;29.

[56] McDevitt M, McDevitt M, Alessandro R, Carlos HV, Jason PH, Shanna RS, et al. Imaging and treating tumor vasculature with targeted radiolabeled carbon nanotubes. International Journal of Nanomedicine 2010.

[57] Avila-Rodriguez MA, Selwyn RG, Hampel JA, Thomadsen BR, DeJesus OT, Converse AK, et al. Positronemitting resin microspheres as surrogates of $90 \mathrm{Y} \mathrm{SIR-Spheres:} \mathrm{a} \mathrm{radiolabeling} \mathrm{and} \mathrm{stability} \mathrm{study.} \mathrm{Nuclear}$ Medicine and Biology 2007;34:585-90.

[58] Zhou M, Melancon M, Stafford RJ, Li J, Nick AM, Tian M, et al. Precision Nanomedicine Using Dual PET and MR Temperature Imaging-Guided Photothermal Therapy. Journal of Nuclear Medicine 2016;57:177883.

[59] Cui L, Xiong C, Zhou M, Shi S, Chow DSL, and Li C. Integrin avß3-Targeted [64Cu]CuS Nanoparticles for PET/CT Imaging and Photothermal Ablation Therapy. Bioconjugate Chemistry 2018;29:4062-71.

[60] Zhou M, Song S, Zhao J, Tian M, and Li C. Theranostic CuS Nanoparticles Targeting Folate Receptors for PET Image-Guided Photothermal Therapy. J Mater Chem B 2015;3:8939-48.

[61] Liu Q, Qian Y, Li P, Zhang S, Wang Z, Liu J, et al. The combined therapeutic effects of 131 iodine-labeled multifunctional copper sulfide-loaded microspheres in treating breast cancer. Acta Pharmaceutica Sinica B 2018;8:371-80.

[62] Yang W, Guo W, Le W, Lv G, Zhang F, Shi L, et al. Albumin-Bioinspired Gd:CuS Nanotheranostic Agent for In Vivo Photoacoustic/Magnetic Resonance Imaging-Guided Tumor-Targeted Photothermal Therapy. ACS Nano 2016;10:10245-57.

[63] Li B, Tang J, Chen W, Hao G, Kurniawan N, Gu Z, et al. Novel theranostic nanoplatform for complete mice tumor elimination via MR imaging-guided acid-enhanced photothermo-/chemo-therapy. Biomaterials 2018;177:40-51.

[64] Fan Q, Cheng K, Hu X, Ma X, Zhang R, Yang M, et al. Transferring Biomarker into Molecular Probe: Melanin Nanoparticle as a Naturally Active Platform for Multimodality Imaging. Journal of the American Chemical Society 2014;136:15185-94.

[65] Zhang R, Fan Q, Yang M, Cheng K, Lu X, Zhang L, et al. Engineering Melanin Nanoparticles as an Efficient Drug-Delivery System for Imaging-Guided Chemotherapy. Advanced Materials 2015;27:5063-9. [66] Yang X, Hong H, Grailer JJ, Rowland IJ, Javadi A, Hurley SA, et al. cRGD-functionalized, DOX-conjugated, and $64 \mathrm{Cu}$-labeled superparamagnetic iron oxide nanoparticles for targeted anticancer drug delivery and PET/MR imaging. Biomaterials 2011;32:4151-60. 
[67] Feng L, Cheng L, Dong Z, Tao D, Barnhart TE, Cai W, et al. Theranostic Liposomes with HypoxiaActivated Prodrug to Effectively Destruct Hypoxic Tumors Post-Photodynamic Therapy. ACS Nano 2017;11:927-37.

[68] Xue X, Huang Y, Bo R, Jia B, Wu H, Yuan Y, et al. Trojan Horse nanotheranostics with dual transformability and multifunctionality for highly effective cancer treatment. Nat Commun 2018;9:3653. [69] Yu B, Goel S, Ni D, Ellison PA, Siamof CM, Jiang D, et al. Reassembly of 89

Zr-Labeled Cancer Cell Membranes into Multicompartment Membrane-Derived Liposomes for PETTrackable Tumor-Targeted Theranostics. Advanced Materials 2018;30. 